\title{
An ALMA Survey of Protoplanetary Disks in the $\sigma$ Orionis Cluster
}

\author{
M. Ansdell ${ }^{1}$, J. P. Williams ${ }^{1}$, C. F. Manara ${ }^{2}$, A. Miotello ${ }^{3}$, S. Facchini ${ }^{4}$, N. van der Marel ${ }^{1}$, L. Testi ${ }^{5,6}$, and E. F. van Dishoeck ${ }^{3,4}$ \\ ${ }^{1}$ Institute for Astronomy, University of Hawai 'i at Mānoa, Honolulu, HI, USA \\ ${ }^{2}$ Scientific Support Office, Directorate of Science, European Space Research and Technology Centre (ESA/ESTEC), \\ Keplerlaan 1, 2201 AZ Noordwijk, The Netherlands \\ ${ }^{3}$ Leiden Observatory, Leiden University, P.O. Box 9513, 2300 RA Leiden, The Netherlands \\ ${ }^{4}$ Max-Plank-Institut für Extraterrestrische Physik, Giessenbachstraße 1, D-85748 Garching, Germany \\ ${ }^{5}$ INAF-Osservatorio Astrofisico di Arcetri, Largo E. Fermi 5, I-50125 Firenze, Italy \\ ${ }^{6}$ European Southern Observatory, Karl-Schwarzschild-Str. 2, D-85748 Garching bei München, Germany \\ Received 2017 February 12; revised 2017 March 22; accepted 2017 March 23; published 2017 May 3
}

\begin{abstract}
The $\sigma$ Orionis cluster is important for studying protoplanetary disk evolution, as its intermediate age ( $\sim 3-5 \mathrm{Myr})$ is comparable to the median disk lifetime. We use ALMA to conduct a high-sensitivity survey of dust and gas in 92 protoplanetary disks around $\sigma$ Orionis members with $M_{*} \gtrsim 0.1 M_{\odot}$. Our observations cover the $1.33 \mathrm{~mm}$ continuum and several $\mathrm{CO} J=2-1$ lines: out of 92 sources, we detect 37 in the millimeter continuum and 6 in ${ }^{12} \mathrm{CO}, 3$ in ${ }^{13} \mathrm{CO}$, and none in $\mathrm{C}^{18} \mathrm{O}$. Using the continuum emission to estimate dust mass, we find only 11 disks with $M_{\text {dust }} \gtrsim 10 M_{\oplus}$, indicating that after only a few Myr of evolution most disks lack sufficient dust to form giant planet cores. Stacking the individually undetected continuum sources limits their average dust mass to $5 \times$ lower than that of the faintest detected disk, supporting theoretical models that indicate rapid dissipation once disk clearing begins. Comparing the protoplanetary disk population in $\sigma$ Orionis to those of other star-forming regions supports the steady decline in average dust mass and the steepening of the $M_{\text {dust }}-M_{*}$ relation with age; studying these evolutionary trends can inform the relative importance of different disk processes during key eras of planet formation. External photoevaporation from the central 09 star is influencing disk evolution throughout the region: dust masses clearly decline with decreasing separation from the photoionizing source, and the handful of $\mathrm{CO}$ detections exist at projected separations of $>1.5 \mathrm{pc}$. Collectively, our findings indicate that giant planet formation is inherently rare and/or well underway by a few Myr of age.
\end{abstract}

Key words: accretion, accretion disks - circumstellar matter - planets and satellites: formation protoplanetary disks - stars: pre-main sequence - stars: protostars

Supporting material: machine-readable tables

\section{Introduction}

Planets are thought to form in so-called "protoplanetary" disks around young stars within $\sim 5-10 \mathrm{Myr}$ (Williams \& Cieza 2011). The resulting exoplanet population is diverse, as revealed by the Kepler transit survey (Borucki et al. 2010) and long-term radial velocity (RV) surveys (Howard et al. 2010; Mayor et al. 2011). However, certain trends are emerging; for example, intermediate-mass planets (i.e., "super-Earths" with masses between that of Earth and Neptune) appear to be an order of magnitude more abundant than gas giants (i.e., planets with masses several times that of Jupiter), at least for short orbital periods (Howard et al. 2012; Fressin et al. 2013; Petigura et al. 2013; Gaidos et al. 2016). To identify the origins of these trends, and thus better understand planet formation, we must survey the preceding protoplanetary disks. Indeed, exoplanet population synthesis models indicate that planetary properties and architectures are often dictated by the initial dust and gas content of protoplanetary disks and their subsequent evolution (e.g., Thommes et al. 2008; Mordasini et al. 2012, 2016; Bitsch et al. 2015).

Sub-millimeter and millimeter wavelength surveys are particularly useful for probing the bulk dust and gas content of protoplanetary disks because disk emission at these longer wavelengths can be optically thin. The first (sub-)millimeter surveys of star-forming regions made the initial steps in identifying trends in protoplanetary disk evolution that could potentially explain correlations seen in the exoplanet population (Andrews et al. 2009, 2013; Lee et al. 2011; Williams et al. 2013; Ansdell et al. 2015). Most notably, early surveys of Taurus disks (Natta et al. 2000; Andrews et al. 2013) revealed a positive dependence between disk dust mass $\left(M_{\text {dust }}\right)$ and host star mass $\left(M_{*}\right)$, which could fundamentally explain the correlation between giant planet frequency and stellar mass (Endl et al. 2006; Johnson et al. 2007; Bowler et al. 2010; Bonfils et al. 2013). However, these initial disk surveys were often incomplete and limited by dust-mass sensitivities of a few Earth masses. These constraints meant that it remained unclear whether (sub-)millimeter continuum emission systematically declines with age, reflecting steady disk dispersal and/or grain growth in protoplanetary disks (e.g., Williams 2012). Moreover, none of these initial surveys probed bulk gas mass, as contemporary facilities lacked the sensitivity to detect faint line emission.

Measuring both dust and gas content independently is essential for studying planet formation, as growing dust grains decouple from the gas and evolve differently, yet both components determine what types of planets may form in a disk. However, due to the challenges associated with estimating disk gas masses, the canonical interstellar medium (ISM) gas-to-dust ratio of $\sim 100$ (Bohlin et al. 1978) is often used to infer gas mass from dust mass, requiring an extrapolation of two orders of magnitude. Recent observations suggest that the inherited ISM ratio may actually decrease by an order of magnitude after just a few Myr of evolution 
(Williams \& Best 2014; Ansdell et al. 2016), though these calculations may be underestimated due to carbon depletion (e.g., Miotello et al. 2017). If gas is being depleted in disks (e.g., due to winds), then this may help to explain the lack of gas giants and prevalence of super-Earths seen in the exoplanet population (e.g., Fressin et al. 2013; Gaidos et al. 2016). In this scenario, super-Earths would result when giant planet cores form in gas-depleted disks, prohibiting the cores from rapidly accreting gaseous envelopes (e.g., Lee \& Chiang 2016) as predicted by core accretion theory (e.g., Pollack et al. 1996; Ida \& Lin 2004).

The enhanced sensitivity of the Atacama Large Millimeter/ sub-millimeter Array (ALMA) now allows for efficient surveys of both dust and gas for large samples of protoplanetary disks across star-forming regions spanning the expected disk lifetime ( 1-10 Myr). The first large-scale protoplanetary disk surveys conducted with ALMA include: Ansdell et al. (2016), who carried out a near-complete survey of 89 protoplanetary disks in the young Lupus star-forming region $(\sim 1-3 \mathrm{Myr}$ at $\sim 150 \mathrm{pc}$; Comerón 2008; Alcalá et al. 2014) with continuum and line sensitivities corresponding to $M_{\text {dust }} \sim 0.3 M_{\oplus}$ and $M_{\text {gas }} \sim 1.0$ $M_{\text {Jup }}$, respectively; Barenfeld et al. (2016), who observed 106 disks in the more evolved Upper Sco region ( 5-10 Myr at $145 \mathrm{pc}$; Preibisch et al. 2002; Pecaut et al. 2012) with sensitivities of $M_{\text {dust }} \sim 0.1 M_{\oplus}$; and Pascucci et al. (2016), who surveyed 93 protoplanetary disks in the young Chamaeleon I region ( 2-3 Myr at $160 \mathrm{pc}$; Luhman 2008) with sensitivities of $M_{\text {dust }} \sim 0.2-0.8 M_{\oplus}{ }^{7}$

These ALMA disk surveys are beginning to reveal trends in protoplanetary disk evolution that can help to constrain planet formation theory and explain correlations seen in the exoplanet population. One of their clearest findings is that average disk dust mass does indeed decrease with age. Ansdell et al. (2016) showed that Lupus disks have a mean dust mass $\sim 3 \times$ higher than that of the older Upper Sco region, but are statistically indistinguishable from disks in the similarly aged Taurus region. Equivalently, Barenfeld et al. (2016) found that the average $M_{\text {dust }} / M_{*}$ ratio in Upper Sco is $\sim 4.5 \times$ lower than in Taurus. Interestingly, even in the younger regions, most disks lack sufficient dust to form the solid cores needed to build gas giants (e.g., only $26 \%$ of protoplanetary disks in Lupus have $M_{\text {dust }} \gtrsim 10 M_{\oplus}$; Ansdell et al. 2016). These findings point to significant global disk evolution during the first few Myr and imply that giant planet formation occurs rapidly and/or is rare.

Moreover, these ALMA disk surveys have confirmed the aforementioned $M_{\mathrm{dust}}-M_{*}$ relation initially seen in the preALMA surveys of Taurus, while also revealing a steepening of the relation with age (Ansdell et al. 2016; Pascucci et al. 2016). This steepening would indicate that dust evolution occurs more rapidly around lower-mass stars, and can be compared to theoretical models to constrain the relative importance of different disk evolution processes (Pascucci et al. 2016). Finally, Manara et al. (2016) have combined estimates of disk mass $\left(M_{\text {disk }}\right)$ from ALMA with spectroscopic measurements of stellar mass accretion rates $\left(\dot{M}_{\text {acc }}\right)$ from VLT/X-Shooter in the Lupus star-forming region to provide the first observational confirmation of the $M_{\mathrm{disk}}-\dot{M}_{\text {acc }}$ relation predicted by viscous disk evolution theory.

\footnotetext{
7 These dust-mass sensitivities correspond to $3 \times$ the $\mathrm{rms}$ and use the assumptions described in Section 5.1 to convert (sub-)millimeter flux to dust mass.
}

The $\sigma$ Orionis region (Walter et al. 2008) is a particularly important target for studying disk evolution due to its intermediate age of 3-5 Myr (Oliveira et al. 2002, 2004), which is comparable to the median disk lifetime (Williams \& Cieza 2011). Only one-third of cluster members (92 sources) exhibit strong infrared (IR) excess indicative of a protoplanetary disk (Hernández et al. 2007). Williams et al. (2013) surveyed the region at $850 \mu \mathrm{m}$ with JCMT/SCUBA-2, detecting just 8 disks at $\sim 4 M_{\oplus}$ sensitivity; they also noted the remarkable diversity in the spectral energy distributions (SEDs) of their detections (see their Figure 3), indicating substantial and ongoing disk evolution. By stacking their individually non-detected disks, Williams et al. (2013) found a mean signal of $1.3 \mathrm{mJy}$ at $4 \sigma$ significance, motivating more sensitive follow-up observations of this region.

We therefore use ALMA to conduct a high-sensitivity millimeter wavelength survey of all known protoplanetary disks in $\sigma$ Orionis in both dust and gas. We describe the sample in Section 2 and our ALMA observations in Section 3. The continuum and line measurements are presented in Section 4, then converted to dust and gas masses in Section 5. We interpret our findings within the context of disk evolution in Section 6 by identifying correlations with stellar and cluster properties as well as comparing our results to those found in other star-forming regions. This work is summarized in Section 7.

\section{Sample}

The $\sigma$ Orionis cluster consists of several hundred young stellar objects (YSOs) ranging from brown dwarfs to OB-type stars (see review in Walter et al. 2008). The cluster is named after its brightest member, $\sigma$ Ori, a trapezium-like system whose most massive component is an O9 star. Cluster membership is defined by the Mayrit catalog (Caballero 2008), which identifies 241 stars and brown dwarfs that are located within 30 arcmin of the $\sigma$ Ori system and exhibit known features of youth (X-ray emission, Li $6708 \AA$ absorption, etc.). We adopt a cluster distance of $385 \mathrm{pc}$ based on recent orbital parallax measurements of the $\sigma$ Ori triple system (Schaefer et al. 2016). The low reddening toward this cluster, estimated at $E(B-V) \sim 0.05$ (e.g., Sherry et al. 2008), makes it a valuable site for analyzing the evolution of young stars.

Our sample consists of the 92 YSOs in $\sigma$ Orionis with IR excesses consistent with the presence of a protoplanetary disk. These sources are identified by cross-matching the Class II and transition disk (TD) candidates from the Spitzer survey of Hernández et al. (2007) with the aforementioned Mayrit catalog (Caballero 2008). Both catalogs are expected to be complete down to the brown dwarf limit. Disk classifications are based on the Spitzer/IRAC SED slope, as described in Hernández et al. (2007). We also include in our sample a Class I disk (1153), as it is located near the Spitzer/IRAC color cutoff for Class II disks.

The sources in our sample are presented in Table 1 with their stellar spectral types (SpT) and stellar masses $\left(M_{*}\right)$. Spectral types were primarily taken from the homogenous sample of low-resolution optical spectra analyzed in Hernández et al. (2014), but supplemented with those from mediumresolution VLT/X-Shooter spectra when available from Rigliaco et al. (2012). For the 23 sources that lack spectroscopic information, we estimate their spectral types using an empirical relation between $V-J$ color and stellar spectral 
Table 1

Continuum Properties

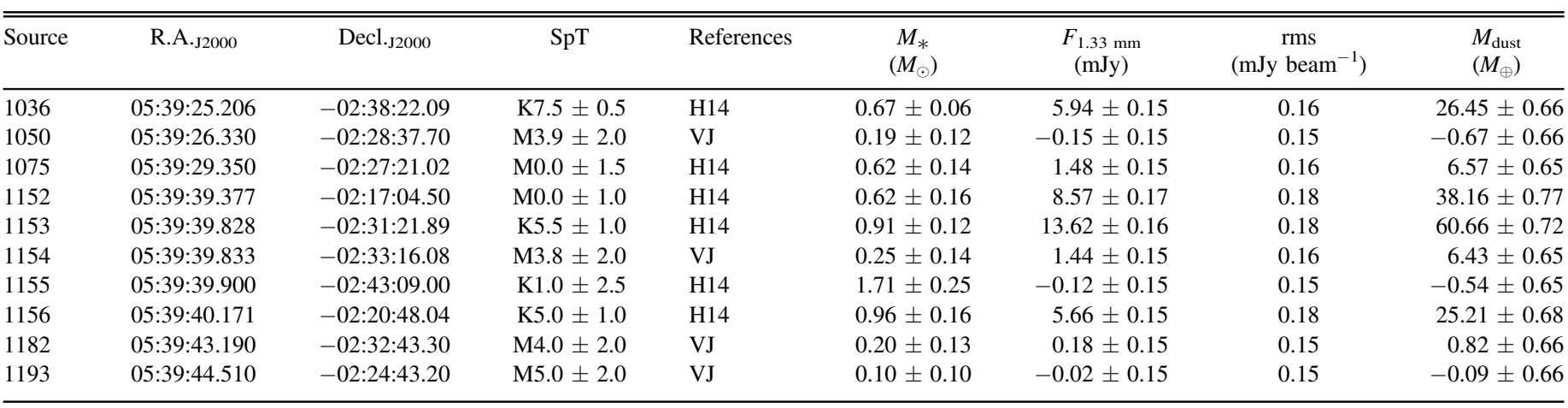

References. H14 = Hernández et al. (2014), R12 = Rigliaco et al. (2012), VJ = derived from $V-J$ colors (see Section 2).

(This table is available in its entirety in machine-readable form.)

type; the relation was derived by measuring synthetic photometry from flux-calibrated VLT/X-Shooter spectra of YSOs with spectral types from G5 to M9.5, then performing a non-parametric fit of the $V-J$ color versus spectral type relation (Manara et al. 2017). For these sources with photometrically derived spectral types, we cautiously assume uncertainties of \pm 2 spectral subtypes. Figure 1 shows the stellar spectral type distribution of our sample.

We estimate $M_{*}$ values for our sample by comparing their positions on the Hertzsprung-Russel (HR) diagram to the evolutionary models of Siess et al. (2000). In order to place our targets on the HR diagram, we convert their spectral types to stellar effective temperatures $\left(T_{\text {eff }}\right)$ and derive their stellar luminosities $\left(L_{*}\right)$ from $J$-band magnitudes using the relations in Herczeg \& Hillenbrand (2015). The uncertainties on $L_{*}$ are obtained by propagating the uncertainties on spectral type and bolometric correction, and thus on distance and optical extinction $\left(A_{V}\right)$. We then calculate the uncertainties on $M_{*}$ using a Monte Carlo (MC) method, where we take the standard deviation of 1000 estimates of $M_{*}$, each calculated after randomly perturbing the derived values of $T_{\text {eff }}$ and $L_{*}$ by their uncertainties.

\section{Alma Observations}

Our Band 6 ALMA observations were obtained on 2016 July 30 and 31 during Cycle 3 (Project ID: 2015.1.00089.S; PI: Williams). The array configuration used 36 and $3712 \mathrm{~m}$ antennas on July 30 and 31, respectively, with baselines of 15-1124 $\mathrm{m}$ on both runs. The correlator setup included two broadband continuum windows centered on 234.293 and $216.484 \mathrm{GHz}$ with bandwidths of 2.000 and $1.875 \mathrm{GHz}$ and channel widths of 15.625 and $0.976 \mathrm{MHz}$, respectively. The bandwidth-weighted mean continuum frequency was $225.676 \mathrm{GHz}(1.33 \mathrm{~mm})$. The spectral windows covered the ${ }^{12} \mathrm{CO} \quad(230.538 \mathrm{GHz}),{ }^{13} \mathrm{CO} \quad(220.399 \mathrm{GHz})$, and $\mathrm{C}^{18} \mathrm{O}$ $(219.560 \mathrm{GHz}) J=2-1$ transitions at velocity resolutions of $0.16-0.17 \mathrm{~km} \mathrm{~s}^{-1}$. These spectral windows were centered on 230.531, 220.392, and $219.554 \mathrm{GHz}$ with bandwidths of 11.719 MHz and channel widths of $0.122 \mathrm{MHz}$.

On-source integration times were 1.2 minutes per object for an average continuum rms of $0.15 \mathrm{mJy}^{\text {beam }}{ }^{-1}$ (Table 1 ). This sensitivity was based on the JCMT/SCUBA-2 survey of $\sigma$ Orionis disks by Williams et al. (2013), who found that stacking their individual non-detections revealed a mean

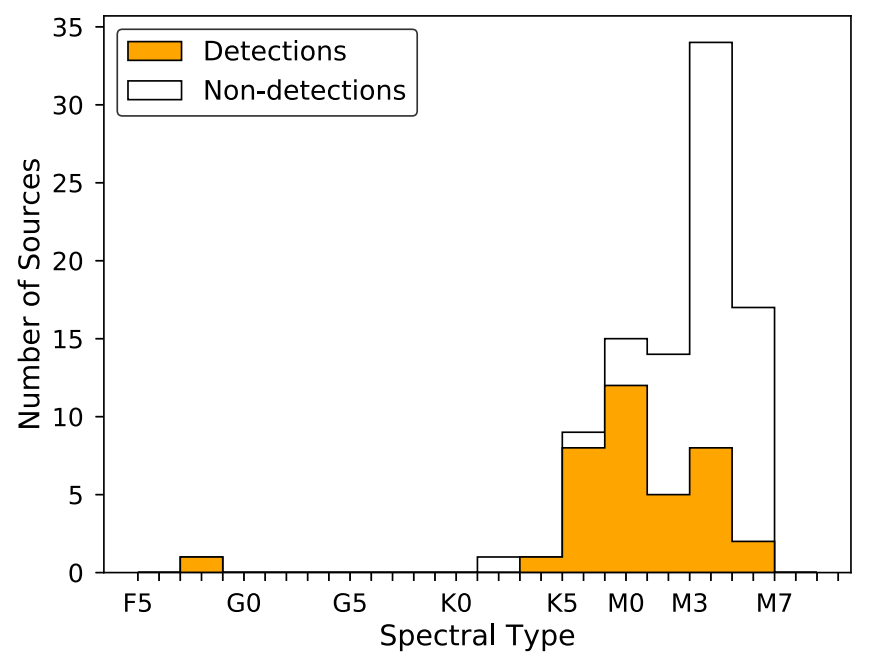

Figure 1. Distribution of stellar spectral types for the sources in $\sigma$ Orionis targeted by our ALMA survey (Table 1). The orange histogram shows continuum detections, while the open histogram shows continuum nondetections (Section 4.1).

$850 \mu \mathrm{m}$ continuum signal of $1.3 \mathrm{mJy}$ at $4 \sigma$ significance. The sensitivity of our ALMA survey was therefore chosen to provide $\sim 3-4 \sigma$ detections of such disks at $1.3 \mathrm{~mm}$, based on an extrapolation of the $850 \mu \mathrm{m}$ mean signal using a spectral slope of $\alpha=2-3$.

The raw data were pipeline calibrated by NRAO staff using the CASA package (version 4.5.3). The pipeline calibration included: absolute flux calibration with observations of J0522-3627 or J0423-0120; bandpass calibration with observations of $\mathrm{J} 0510+1800$ or $\mathrm{J} 0522-3627$; and gain calibration with observations of J0532-0307. We estimate an absolute flux calibration error of $\sim 10 \%$ based on the amplitude variations of gain calibrators over time.

We extract the continuum images from the calibrated visibilities by averaging over the continuum channels and cleaning with a Briggs robust weighting parameter of +0.5 for an average beam size of 0 " $31 \times 0$ !" $25(\sim 120 \times 95$ au at $385 \mathrm{pc})$. We extract ${ }^{12} \mathrm{CO},{ }^{13} \mathrm{CO}$, and $\mathrm{C}^{18} \mathrm{O} J=2-1$ line channel maps from the calibrated visibilities by subtracting the continuum from the spectral windows containing line emission using the uvcontsub routine in CASA. Sources showing clear line emission were cleaned with a Briggs robust weighting parameter of +0.5 . We find average rms values of 13,14 , and 

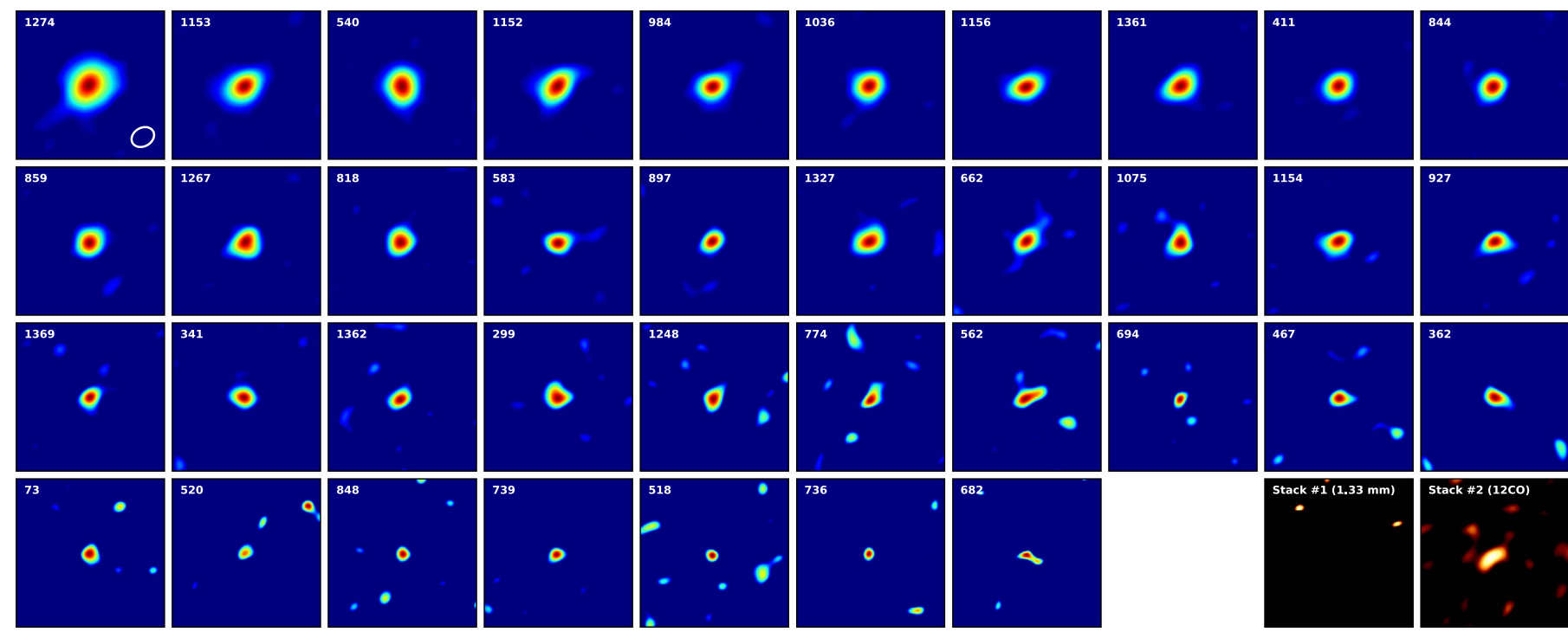

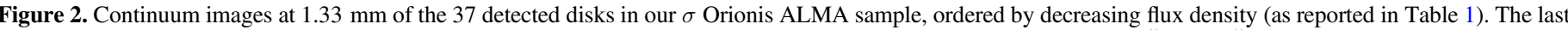

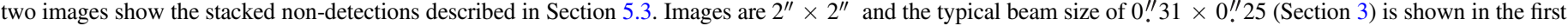
panel.

$11 \mathrm{mJy}$ beam $^{-1}$ within $1 \mathrm{~km} \mathrm{~s}^{-1}$ velocity channels for the ${ }^{12} \mathrm{CO},{ }^{13} \mathrm{CO}$, and $\mathrm{C}^{18} \mathrm{O}$ lines, respectively.

\section{Alma Results}

\subsection{33 mm Continuum Emission}

Nearly all sources in our sample are unresolved, thus we measure continuum flux densities by fitting point-source models to the visibility data using the uvmodelfit routine in CASA. The point-source model has three free parameters: integrated flux density $\left(F_{\lambda}\right)$, right ascension offset from the phase center $(\Delta \alpha)$, and declination offset from the phase center $(\Delta \delta)$. For the five resolved sources $(1036,1152,1153,1274$, and 540), we fit an elliptical Gaussian model instead, which has three additional free parameters: FWHM along the major axis $(a)$, aspect ratio of the axes $(r)$, and position angle (PA). We scale the uncertainties on the fitted parameters by the square root of the reduced $\chi^{2}$ value of the fit.

Table 1 presents the $1.33 \mathrm{~mm}$ continuum flux densities and associated uncertainties $\left(F_{1.33} \mathrm{~mm}\right)$, where the uncertainties are statistical errors and do not include the $10 \%$ absolute flux calibration error (Section 3). We detect only 37 out of the 92 observed sources at $>3 \sigma$ significance (Figure 2). For detections, the source locations in Table 1 are the fitted source centers output by uvmodelfit, while for non-detections they are simply the phase centers of the ALMA observations, which were chosen based on 2MASS positions. The average offsets from the phase centers for the detections are $\Delta \alpha=0$ ". 057 and $\Delta \delta=-0$." 096 (1.9 and -3.2 pixels), both much smaller than the average beam size (Section 3 ). We also note that only 5 out of the 37 continuum detections have photometrically derived spectral types, which are less precise than the spectroscopically determined spectral types (Section 2).

\subsection{CO Line Emission}

To search for objects exhibiting significant line emission, we first extract the ${ }^{12} \mathrm{CO}$ spectrum for each source. When creating the spectrum, we use $1 \mathrm{~km} \mathrm{~s}^{-1}$ velocity sampling and measure fluxes in each channel using a circular aperture 0 ". 30 in radius and centered on the continuum emission (for detections) or the expected stellar position (for non-detections). We measure the image rms using a $4^{\prime \prime}$ to $9^{\prime \prime}$ radius annulus centered on the fitted or expected source position. Candidate detections are identified as those with emission exceeding $3 \times$ the rms in multiple nearby channels within $0-25 \mathrm{~km} \mathrm{~s}^{-1}$ (LSRK frame), which covers the range of RVs found for $\sigma$ Orionis members (e.g., Jeffries et al. 2006).

For each candidate detection, we create zero-moment maps by integrating across the velocity range where the emission exceeds the noise. The integrated flux $\left(F_{12 \mathrm{CO}}\right)$ is then measured using circular aperture photometry, where the aperture radius for each source is determined by a curve-of-growth method in which successively larger apertures are applied until the flux stabilizes to within errors. Uncertainties $\left(E_{12 \mathrm{CO}}\right)$ are estimated by taking the standard deviation of the fluxes measured within the same sized aperture placed randomly within the field of view but away from the source. We consider sources as detections when $F_{12 \mathrm{CO}}>4 \times E_{12 \mathrm{CO}}$. We adopt this high detection threshold because, as pointed out in Barenfeld et al. (2016), this procedure selects both the velocity range and aperture size that maximize the signal, thus can produce false detections at lower significance levels. We detect only six sources in ${ }^{12} \mathrm{CO}$ using this procedure. For these sources, we also search for ${ }^{13} \mathrm{CO}$ and $\mathrm{C}^{18} \mathrm{O}$ emission using the same velocity range and aperture photometry method as for ${ }^{12} \mathrm{CO}$; we detect three of these sources in ${ }^{13} \mathrm{CO}$ and none in $\mathrm{C}^{18} \mathrm{O}$.

For sources with no significant line emission found using the above procedure, we create zero-moment maps by integrating across the channels $\pm 1 \mathrm{~km} \mathrm{~s}^{-1}$ from their known RVs, when available in the literature (Maxted et al. 2008; Sacco et al. 2008). For sources with unknown RVs, we integrate around the average value for $\sigma$ Orionis members with known RVs. Jeffries et al. (2006) showed that $\sigma$ Orionis members are divided into two kinematically distinct subgroups differentiated by their RVs (Group 1 and 2, by their convention). However, the region is dominated by Group 2 sources at $\delta<-02: 18: 00$, where all our gas non-detections with unknown RVs are located. Thus we adopt the average RV of Group $2\left(13 \mathrm{~km} \mathrm{~s}^{-1}\right.$ 
Table 2

Gas Properties

\begin{tabular}{|c|c|c|c|c|c|c|}
\hline Source & $\begin{array}{c}F_{12 \mathrm{CO}} \\
\left(\mathrm{mJy} \mathrm{km} \mathrm{s}^{-1}\right)\end{array}$ & $\begin{array}{c}F_{13 \mathrm{CO}} \\
\left(\mathrm{mJy} \mathrm{km} \mathrm{s}^{-1}\right)\end{array}$ & $\begin{array}{c}F_{\mathrm{C} 18 \mathrm{O}} \\
\left(\mathrm{mJy} \mathrm{km} \mathrm{s}^{-1}\right)\end{array}$ & $\begin{array}{c}M_{\text {gas }} \\
\left(M_{\text {Jup }}\right)\end{array}$ & $\begin{array}{c}M_{\text {gas,min }} \\
\left(M_{\text {Jup }}\right)\end{array}$ & $\begin{array}{c}M_{\text {gas,max }} \\
\left(M_{\text {Jup }}\right)\end{array}$ \\
\hline \multicolumn{7}{|c|}{ Gas Detections } \\
\hline 1274 & $861 \pm 88$ & $326 \pm 68$ & $<48$ & 5.5 & 1.0 & 31.4 \\
\hline 1152 & $633 \pm 82$ & $314 \pm 65$ & $<60$ & 7.1 & 1.0 & 31.4 \\
\hline 1153 & $557 \pm 57$ & $<99.0$ & $<72$ & $\ldots$ & $\ldots$ & 1.0 \\
\hline \multicolumn{7}{|c|}{ Gas Non-detections } \\
\hline 1036 & $<72.0$ & $<81.0$ & $<57$ & $\ldots$ & $\ldots$ & 3.1 \\
\hline 1050 & $<72.0$ & $<81.0$ & $<60$ & $\ldots$ & $\ldots$ & 3.1 \\
\hline 1154 & $<69.0$ & $<75.0$ & $<57$ & $\ldots$ & $\ldots$ & 3.1 \\
\hline 1155 & $<69.0$ & $<78.0$ & $<57$ & $\ldots$ & $\ldots$ & 3.1 \\
\hline 1248 & $<72.0$ & $<81.0$ & $<60$ & $\ldots$ & $\ldots$ & 3.1 \\
\hline 1260 & $<69.0$ & $<78.0$ & $<60$ & $\ldots$ & $\ldots$ & 3.1 \\
\hline
\end{tabular}

(This table is available in its entirety in machine-readable form.)

in the LSRK frame) when creating zero-moment maps for our gas non-detections with unknown RVs. We measure ${ }^{12} \mathrm{CO}$, ${ }^{13} \mathrm{CO}$, and $\mathrm{C}^{18} \mathrm{O}$ integrated fluxes from these zero-moment maps using the aforementioned aperture photometry method, but with an aperture size fixed to the beam size. We found no additional detections, and thus took upper limits as $3 \times$ the image rms.

Table 2 gives our integrated line fluxes or upper limits. Of the 92 targets, only 6 are detected in ${ }^{12} \mathrm{CO}, 3$ are detected in ${ }^{13} \mathrm{CO}$, and none are detected in $\mathrm{C}^{18} \mathrm{O}$ with $>4 \sigma$ significance. All sources detected in ${ }^{12} \mathrm{CO}$ are detected in the continuum, and all sources detected in ${ }^{13} \mathrm{CO}$ are detected in ${ }^{12} \mathrm{CO}$. The zeroand first-moment maps of the gas detections are shown in Figure 3.

\section{Properties of $\sigma$ Orionis Disks}

\subsection{Dust Masses}

Because dust emission at (sub-)millimeter wavelengths can be optically thin, the bulk dust mass of a disk ( $\left.M_{\text {dust }}\right)$ can be estimated from its (sub-)millimeter continuum emission at a given wavelength $\left(F_{\nu}\right)$, as shown in Hildebrand (1983):

$$
M_{\text {dust }}=\frac{F_{\nu} d^{2}}{\kappa_{\nu} B_{\nu}\left(T_{\text {dust }}\right)} .
$$

where $B_{\nu}\left(T_{\text {dust }}\right)$ is the Planck function for a characteristic dust temperature of $T_{\text {dust }}=20 \mathrm{~K}$ (the median for Taurus disks; Andrews \& Williams 2005). We take the dust grain opacity, $\kappa_{\nu}$, as $10 \mathrm{~cm}^{2} \mathrm{~g}^{-1}$ at $1000 \mathrm{GHz}$ and use an opacity power-law index of $\beta=1$ (Beckwith et al. 1990). The source distance, $d$, is taken as $385 \mathrm{pc}$ based on the updated parallax of the $\sigma$ Ori triple system (Schaefer et al. 2016). Equation (1) can therefore be approximated as $M_{\text {dust }} \approx 1.34 \times 10^{-5} F_{1.33 \mathrm{~mm}}$, where $F_{1.33 \mathrm{~mm}}$ is in $\mathrm{mJy}$ and $M_{\text {dust }}$ is in $M_{\odot}$.

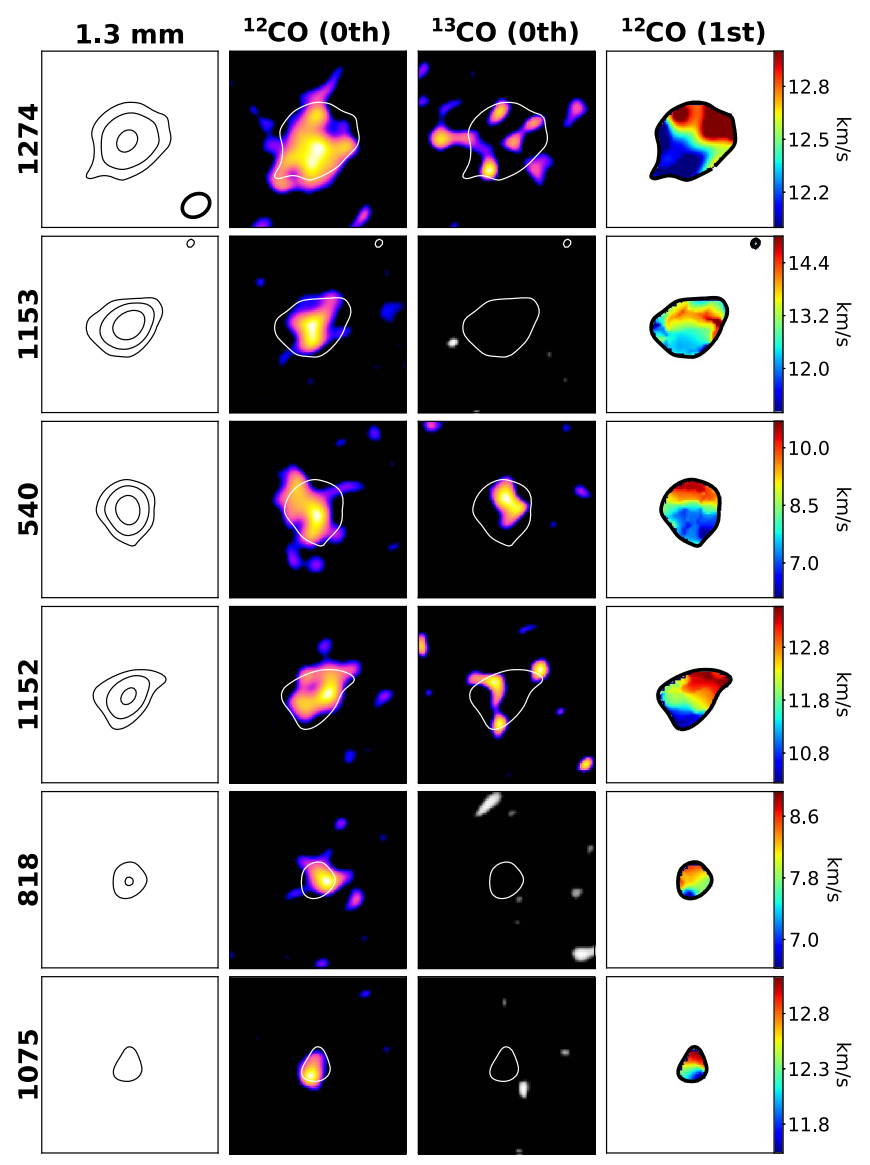

Figure 3. The six sources in our sample detected in $\mathrm{CO}$ (Section 4.2). The first column shows the $1.33 \mathrm{~mm}$ continuum emission in $4 \sigma, 10 \sigma$, and $25 \sigma$ contours. The second and third columns show the ${ }^{12} \mathrm{CO}$ and ${ }^{13} \mathrm{CO}$ zero-moment maps with $4 \sigma$ continuum contours. The last column shows the ${ }^{12} \mathrm{CO}$ first-moment maps within $4 \sigma$ continuum contours. Images are $2^{\prime \prime} \times 2^{\prime \prime}$ and the typical beam size is given in the first panel. 


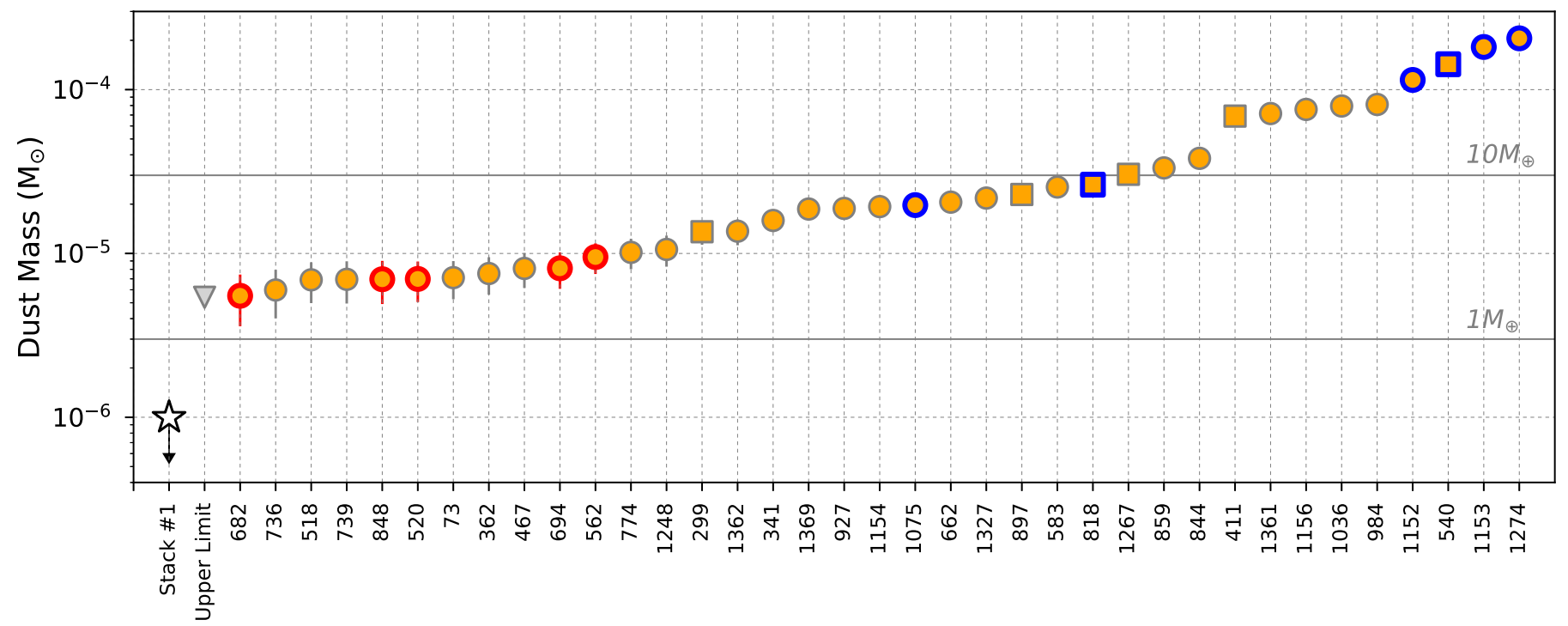

Figure 4. Dust masses for the 37 continuum-detected sources in our $\sigma$ Orionis ALMA sample. Dust masses are from Table 1 and error bars include the 10\% absolute flux calibration uncertainty (Section 3). The downward-facing triangle is the typical $3 \sigma$ upper limit for individual continuum non-detections, while the star shows the constraint on their average dust mass from the stacked continuum non-detections ("Stack \#1" in Section 5.3). Squares indicate TDs identified by their SEDs (Section 5.4). Sources outlined in blue are also detected in ${ }^{12} \mathrm{CO}$ (Section 5.2) and sources outlined in red are $\lesssim 0.5 \mathrm{pc}$ from the central OB system (Section 6.1); sources closest to the central OB system have the lowest dust masses, while sources with the highest dust masses tend to also be detected in CO.

We use this simplified approach, which assumes a single grain opacity and an isothermal disk temperature, to ease comparisons with other disk surveys (Sections 6.2 and 6.3). Moreover, although Andrews et al. (2013) derived a $T_{\text {dust }}=25 \mathrm{~K} \times\left(L_{*} / L_{\odot}\right)^{0.25}$ relation from two-dimensional continuum radiative transfer models, we use an isothermal disk temperature because more detailed modeling of resolved disks suggests that $T_{\text {dust }}$ is independent of stellar parameters. In particular, Tazzari et al. (2017) fit continuum observations of 36 resolved disks in Lupus directly in the $u v$-plane to two-layer disk models by solving energy balance equations at each disk radius, finding no dependence of $T_{\text {dust }}$ as a function of stellar parameters.

Table 1 presents our $M_{\text {dust }}$ estimates, derived using Equation (1) with our $F_{1.33 \mathrm{~mm}}$ measurements (Section 4.1 ). Figure 4 shows the continuum-detected disks in order of increasing $M_{\text {dust }}$ as well as the typical $3 \sigma$ upper limit of $\sim 2.0 M_{\oplus}\left(F_{1.33 \mathrm{~mm}} \sim 0.45 \mathrm{mJy}\right)$. Only four disks have $M_{\text {dust }}>30 M_{\oplus}\left(F_{1.33 \mathrm{~mm}}>6.7 \mathrm{mJy}\right)$, thus nearly all protoplanetary disks in $\sigma$ Orionis have dust masses well below the minimum mass of solids needed to form the planets in our solar system (Weidenschilling 1977). Moreover, only 11 disks have $M_{\text {dust }}>10 M_{\oplus}\left(F_{1.33 \mathrm{~mm}}>2.2 \mathrm{mJy}\right)$, thus by 3-5 Myr most protoplanetary disks appear to lack sufficient dust to form giant planet cores. Note that significant amounts of solids may still exist in objects greater than a few centimeters in size because these larger solids do not produce detectable (sub-) millimeter emission.

\subsection{Gas Masses}

CO line emission can be used to roughly estimate bulk gas masses $\left(M_{\mathrm{gas}}\right)$ independently from the dust, assuming simple $\mathrm{CO}$ chemistry and adopting an ISM-like $\mathrm{CO} / \mathrm{H}_{2}$ abundance. Williams \& Best (2014; hereafter WB14) used parametrized gas disk models to demonstrate that the majority of CO may exist in the warm molecular layer, where it is sufficiently warm to survive freeze-out onto the disk midplane as well as adequately shielded from UV radiation to avoid photodissociation (except for particularly cold or low-mass disks). The CO isotopologue lines, specifically ${ }^{13} \mathrm{CO}$ and $\mathrm{C}^{18} \mathrm{O}$, are especially useful for constraining $M_{\text {gas }}$ as their moderate-to-low optical depths mean that they trace the bulk gas content rather than the temperature profile of the disk. Ansdell et al. (2016) and Miotello et al. (2017) have used these $\mathrm{CO}$ isotopologue lines to estimate $M_{\text {gas }}$ for protoplanetary disks in the Lupus clouds that have been surveyed by ALMA.

Unfortunately, because we find no $\mathrm{C}^{18} \mathrm{O}$ detections in $\sigma$ Orionis, we cannot use the same combination of $\mathrm{CO}$ isotopologue lines to estimate $M_{\mathrm{gas}}$ in this region. However, we can still place rough constraints on $M_{\text {gas }}$ by comparing our measured ${ }^{12} \mathrm{CO}$ and ${ }^{13} \mathrm{CO}$ line luminosities or upper limits to the WB14 model grid. The uncertainties on $M_{\text {gas }}$ are larger for this line combination because ${ }^{12} \mathrm{CO}$ is optically thick and therefore more sensitive to other disk parameters, such as the temperature profile. Nevertheless, $M_{\text {gas }}$ can still be estimated using this method because the combination of integrated line fluxes still primarily depends on bulk gas mass rather than these other disk parameters (see the parameter exploration described in WB14 as well as the separation of gas masses in Figure 5).

Figure 5 compares the WB14 model grid to our measured ${ }^{12} \mathrm{CO}$ and ${ }^{13} \mathrm{CO}$ line luminosities for sources detected in at least one of these lines. Table 2 provides our $M_{\text {gas }}$ constraints derived from the WB14 model grid. To estimate $M_{\text {gas }}$ for the three sources detected in both ${ }^{12} \mathrm{CO}$ and ${ }^{13} \mathrm{CO}(540,1274$, and 1152 ), we calculate the mean (in log space) of the WB14 model grid points consistent with our measured line luminosities and their associated errors; these values span 2-7 $M_{\text {Jup }}$. We also set upper $\left(M_{\mathrm{gas}, \max }\right)$ and lower $\left(M_{\mathrm{gas}, \mathrm{min}}\right)$ limits based on the maximum and minimum WB14 model grid points consistent with the data, respectively. For the three sources with ${ }^{12} \mathrm{CO}$ detections and ${ }^{13} \mathrm{CO}$ upper limits $(1153,818$, and 1075), we provide only $M_{\mathrm{gas}, \mathrm{max}}$, since the lower bound is constrained by the limits of the WB14 model grid, and at these very low masses photodissociation becomes important and self-shielding needs to be taken into account. For the 86 sources undetected in 


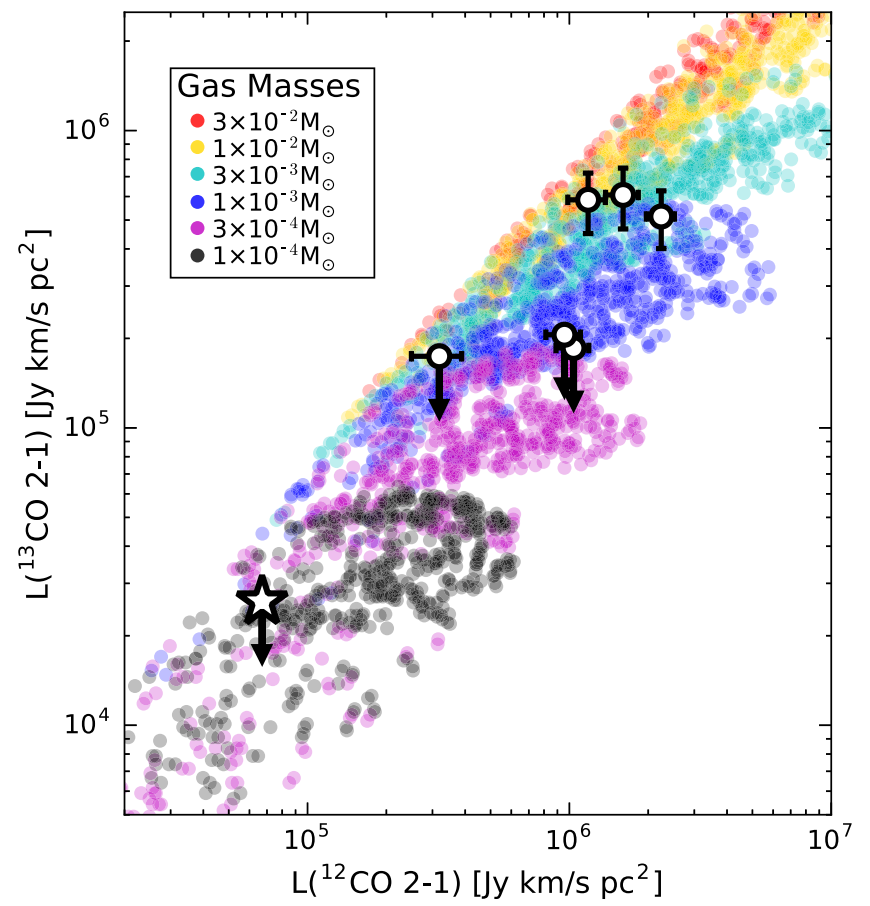

Figure 5. ${ }^{12} \mathrm{CO}$ and ${ }^{13} \mathrm{CO} J=1-2$ line luminosities for determining gas masses (Section 5.2). Colored points show the WB14 model grid color-coded by gas mass. The three disks with both lines detected are plotted as white circles, and the three disks with only ${ }^{12} \mathrm{CO}$ detections are plotted as white circles with arrows indicating $3 \sigma$ upper limits on ${ }^{13} \mathrm{CO}$. Error bars account for the statistical errors given in Table 2 as well as the $10 \%$ absolute flux calibration error. The star shows the location of "Stack \#2" (Section 5.3), where the error bars are smaller than the symbol.

both lines, we give only $M_{\text {gas,max }}$, which is set by the maximum WB14 model grid point consistent with the upper limits on both lines.

Alternatively, one can estimate $M_{\text {gas }}$ by employing the grid of physical-chemical models presented in Miotello et al. (2016; hereafter M16). Using the code DALI (Bruderer et al. 2012), M16 investigated a range of realistic disk and stellar parameters, calculating self-consistently the thermal and chemical structure of the disk up to an age of 1 Myr. As shown by Miotello et al. (2017) for Lupus disks, the simulated line fluxes can be used to derive $M_{\text {gas }}$ from individual ${ }^{13} \mathrm{CO}$ or $\mathrm{C}^{18} \mathrm{O}$ isotopologue line observations, assuming an abundance of volatile carbon. This is because the medians of the simulated line luminosities can be fit by simple functions of $M_{\text {gas }}$ (see Equation (2) in M16). Namely, for low-mass disks, the dependence of the line luminosities on $M_{\text {gas }}$ is linear, while the trend becomes logarithmic for more massive disks due to the line emission becoming optically thick. Thus, the observed ${ }^{13} \mathrm{CO}$ or $\mathrm{C}^{18} \mathrm{O}$ line luminosities directly trace $M_{\text {gas }}$ only if they fall in the linear dependence regime found in M16.

Applying the methodology of M16 to our $\sigma$ Orionis sample, we find that the ${ }^{13} \mathrm{CO}$ upper limits for the three sources detected only in ${ }^{12} \mathrm{CO}(1153,818$, and 1075$)$ are in the linear dependence regime and thus provide upper limits on $M_{\mathrm{gas}}$. Although the three brightest disks (540, 1274, and 1152) have ${ }^{13} \mathrm{CO}$ line luminosities that fall in the logarithmic dependence regime, their $\mathrm{C}^{18} \mathrm{O}$ upper limits can still provide constraints on $M_{\text {gas }}$. The derived $M_{\text {gas }}$ upper limits and the $\mathrm{CO}$ isotopologue lines used for the calculations are reported in Table 3 . These
Table 3

Gas Properties Derived from Miotello et al. (2016) Models

\begin{tabular}{lccc}
\hline \hline Source & $M_{\text {gas }}\left(M_{\text {Jup }}\right)$ & Gas-to-dust Ratio & Line \\
\hline 540 & $<5.8$ & $<41$ & $\mathrm{C}^{18} \mathrm{O}$ \\
1274 & $<3.1$ & $<15$ & $\mathrm{C}^{18} \mathrm{O}$ \\
1152 & $<4.2$ & $<37$ & $\mathrm{C}^{18} \mathrm{O}$ \\
1153 & $<0.3$ & $<2$ & ${ }^{13} \mathrm{CO}$ \\
818 & $<0.4$ & $<15$ & ${ }^{13} \mathrm{CO}$ \\
1075 & $<0.3$ & $<15$ & ${ }^{13} \mathrm{CO}$ \\
\hline
\end{tabular}

results place stronger constraints on $M_{\text {gas }}$ than the WB14 model grid for all six sources detected in $\mathrm{CO}$.

There are several caveats to our derived gas masses. Namely, these gas masses depend inversely on the assumed molecular CO abundance of $[\mathrm{CO}] /\left[\mathrm{H}_{2}\right]=10^{-4}$ in the case of WB14, and the assumed volatile carbon abundance of $[\mathrm{C}] /[\mathrm{H}]=1.35 \times 10^{-4}$ and chemical age of $1 \mathrm{Myr}$ in the case of M16. Additionally, both WB14 and M16 assume an isotopologue ratio of $[\mathrm{CO}] /\left[{ }^{13} \mathrm{CO}\right]=$ 70. The WB14 $[\mathrm{CO}] /\left[\mathrm{H}_{2}\right]$ abundance is consistent with those measured in molecular clouds (Frerking et al. 1982; Lacy et al. 1994; Ripple et al. 2013; Shimajiri et al. 2014) as well as with a direct measurement in a disk (France et al. 2014). However, the strong HD (Bergin et al. 2013) but weak $\mathrm{C}^{18} \mathrm{O}$ emission toward the TW Hydra disk has been interpreted as resulting from significant carbon depletion of up to two orders of magnitude in this system (Favre et al. 2013; Kama et al. 2016; Schwarz et al. 2016).

If carbon depletion (rather than gas depletion) is the true cause of weak $\mathrm{CO}$ emission, the responsible physical mechanisms are not yet established. One hypothesis is that gas-phase reactions initiated by X-ray and cosmic-ray ionization of $\mathrm{He}$ produce $\mathrm{He}^{+}$atoms that react with gaseous $\mathrm{CO}$ to gradually extract carbon, which is then processed into more complex molecules that freeze onto cold dust grains at higher temperatures than CO (Aikawa et al. 1997; Bruderer et al. 2012; Favre et al. 2013; Bergin et al. 2014; Kama et al. 2016; Yu et al. 2016). Alternatively, CO can be turned into more complex organics such as $\mathrm{CH}_{3} \mathrm{OH}$, or into $\mathrm{CO}_{2}$ and $\mathrm{CH}_{4}$, via ice chemistry reactions (e.g., see Figure 3(c) in Eistrup et al. 2016); these reactions have typical timescales of a few Myr (depending on the ionization rate) and thus could be more significant in older systems. Finally, volatile carbon may be locked up in large icy bodies in the disk midplane (Bergin et al. 2010; Ros \& Johansen 2013; Guidi et al. 2016). These large pebbles cannot diffuse upward and thus would no longer participate in gas-phase chemistry (Du et al. 2015; Kama et al. 2016). Such a process would "dry out" the CO from the warm molecular layer, analogous to what is proposed to explain the under-abundance of gas-phase water in disk atmospheres (Bergin et al. 2010; Hogerheijde et al. 2011). If any of these mechanisms significantly depletes carbon in the disk, our derived gas masses would be underestimated.

\subsection{Stacking Analysis}

We perform a stacking analysis to constrain the average dust and gas masses of the individually undetected sources in our sample. To stack the images, we average them in the image plane after centering them on their expected source locations. We then search for emission using the aperture photometry method described in Section 4.2. 
We first stack the 55 sources undetected in the continuum ("Stack \#1"), but do not find a significant mean signal in the continuum or any of the $\mathrm{CO}$ lines. The measured continuum mean signal is $0.05 \pm 0.03 \mathrm{mJy}(1.7 \sigma)$. We confirm this nondetection by calculating the mean and standard error on the mean from the continuum fluxes reported in Table 1, which similarly gives $0.03 \pm 0.02 \mathrm{mJy}(1.5 \sigma)$. This provides a $3 \sigma$ upper limit on the average dust mass of individually undetected continuum sources of $0.4 M_{\oplus}$, which is $5 \times$ lower than the smallest dust mass among the continuum-detected sources in $\sigma$ Orionis (see Figure 4). This striking difference in the dust masses of detected and undetected continuum sources was also seen in an ALMA survey of Lupus disks (see Figure 3 in Ansdell et al. 2016) and further supports theoretical models that predict protoplanetary disks dispersing rapidly once disk clearing begins (e.g., see the review in Alexander et al. 2014).

We also stack the 31 sources that are detected in the continuum but undetected in ${ }^{12} \mathrm{CO}$ ("Stack \#2"), finding a mean continuum signal of $2.29 \pm 0.09 \mathrm{mJy}$ as well as a significant mean ${ }^{12} \mathrm{CO}$ signal of $36 \pm 8 \mathrm{mJy} \mathrm{km} \mathrm{s}^{-1}(4.5 \sigma)$. No emission is detected in the ${ }^{13} \mathrm{CO}$ or $\mathrm{C}^{18} \mathrm{O}$ lines with $3 \sigma$ upper limits of 14 and $11 \mathrm{mJy} \mathrm{km} \mathrm{s}^{-1}$, respectively. The continuum flux corresponds to a dust mass of $\sim 10 M_{\oplus}$, while the ${ }^{12} \mathrm{CO}$ detection and ${ }^{13} \mathrm{CO}$ upper limit correspond to a gas mass $<1.0 M_{\text {Jup }}$ using the WB14 model grid (the ${ }^{13} \mathrm{CO}$ and $\mathrm{C}^{18} \mathrm{O}$ upper limits also correspond to a gas mass $\left.<1.0 M_{\mathrm{Jup}}\right)$. This gives an average gas-to-dust ratio of $<30$ for sources detected in the continuum but not in $\mathrm{CO}$, assuming standard $\mathrm{CO}$ abundances.

\subsection{Transition Disks}

TDs are protoplanetary disks with large inner cavities in their dust distributions (see the review in Espaillat et al. 2014). TDs can be identified by resolved (sub-)millimeter images, or by the mid-IR deficits in their SEDs, which indicate a lack of warm micron-sized dust grains close to the central star. Eight $\sigma$ Orionis members $(1268,1267,908,897,818,540,411$, and 299) have been identified as TDs based on their SEDs (Hernández et al. 2007; Maucó et al. 2016) and all of these sources are included in our ALMA sample.

Six of these TDs $(1267,897,818,540,411$, and 299) were detected by our ALMA continuum observations, and two of the continuum-detected sources (818 and 540) were also detected in CO. Our ALMA observations did not resolve any dust cavities (see Figure 2), though this is not particularly surprising given the large beam size of our observations $(\sim 120 \times 95$ au at 385 pc; Section 3).

Thus $16 \%(6 / 37)$ of our ALMA continuum detections are TDs (see Figure 4), which is interestingly similar to the $19 \%$ fraction found in Lupus (Ansdell et al. 2016) and consistent with previous findings that TDs tend to be among the brightest disks in a given star-forming region (Andrews et al. 2011; Ansdell et al. 2016). Moreover, the TD fraction across the entire protoplanetary disk population in $\sigma$ Orionis is $8 \%(8 / 92)$, which is consistent with TD fractions found in other star-forming regions (see Figure 11 in Espaillat et al. 2014).

\section{Discussion}

\subsection{External UV Photoevaporation from $\sigma$ Ori}

OB associations host at their centers very massive stars with $\mathrm{O}$ - and B-type spectral classifications, surrounded by populations of several hundred to thousands of lower-mass stars. The massive OB stars emit large numbers of extreme-ultraviolet (EUV; $h \nu>13.6 \mathrm{eV}$ ) and far-ultraviolet (FUV; $6 \mathrm{eV}<h \nu<13.6 \mathrm{eV}$ ) photons, which can heat and photoevaporate circumstellar disks around the nearby low-mass stars. The resulting mass-loss rate for a given disk depends on the relative strengths of the incident FUV and EUV fluxes, and thus should depend on the distance of the source from the central OB stars (e.g., Johnstone et al. 1998; Störzer \& Hollenbach 1999).

Mann et al. (2014) used ALMA to search for observational evidence of these external photoevaporation effects on proplyds in the young $(\sim 1-2 \mathrm{Myr})$ Orion Nebula Cluster (ONC). They found a clear drop in disk mass at small projected separations from the central O6 star, $\theta^{1}$ Ori C. Namely, there was a lack of massive $\left(M_{\text {dust }} \gtrsim 9 M_{\oplus}\right)$ disks within $\sim 0.03 \mathrm{pc}$ of $\theta^{1}$ Ori C, where models predict that EUV emission dominates the radiation field (Johnstone et al. 1998). At larger separations of $\sim 0.03-0.3 \mathrm{pc}$, where less energetic FUV emission is expected to dominate (Adams et al. 2004), they found a range of disk masses representative of typical low-mass star-forming regions, indicating that the lower mass-loss rates in FUVdominated regions can preserve disk masses for up to a couple Myr. Mann et al. (2014) concluded that planet formation is likely inhibited for disks in the inner-most EUV-dominated regions of $\mathrm{OB}$ associations due to higher mass-loss rates, while disks in the FUV-dominated regions and beyond are relatively unaffected with planet formation proceeding as in isolated disks.

Mann et al. (2015) looked for similar effects in the very young NGC 2024 region, which at $\sim 0.5$ Myr old (e.g., Levine et al. 2006) hosts the massive star IRS 2b of O8-B2 spectral type (Bik et al. 2003) as well as several hundred YSOs still heavily embedded in molecular cloud material. Mann et al. (2015) could not identify a distance-dependent disk mass distribution in NGC 2024, and instead found several massive $\left(M_{\text {dust }} \gtrsim 17 M_{\oplus}\right)$ disks located $<0.01$ pc from IRS $2 \mathrm{~b}$. They argued that this could be an evolutionary effect: the extremely young age of NGC 2024 simply means that processes like external photoevaporation have not yet had time to significantly reduce disk masses. Alternatively, they suggested that this could be an environmental outcome: the significant cloud material in NGC 2024 may efficiently absorb the high-energy photons from IRS $2 b$, or the later spectral type of the star (compared to $\theta^{1}$ Ori $\mathrm{C}$ in the $\mathrm{ONC}$ ) means that it does not produce sufficient photoionizing radiation.

Here we search for evidence of external photoevaporation in $\sigma$ Orionis, an OB association whose central trapezium system, $\sigma$ Ori, contains a massive O9 star. $\sigma$ Orionis is an interesting target for studying external photoevaporation, as its lack of cloud material and older age may both enhance the observable effects of external photoevaporation. Figure 6 (upper right panel) plots disk dust mass as a function of projected separation from $\sigma$ Ori $(\alpha=05: 38: 44.779, \delta=-02: 36: 00.11)$. Similar to the $\mathrm{ONC}$, we find a lack of massive $\left(M_{\text {dust }} \gtrsim 3 M_{\oplus}\right)$ disks close to the central $\mathrm{OB}$ system; however, the drop in occurrence is seen at $\sim 0.5 \mathrm{pc}-$ a much larger projected distance compared to the $\sim 0.03$ pc limit found for the ONC (Mann et al. 2014). Moreover, beyond $\sim 0.5 \mathrm{pc}$, we see a smooth distancedependent dust-mass distribution that extends out to several parsecs. We note that the smaller dust masses and larger projected distances found in $\sigma$ Orionis (compared to those found in the $\mathrm{ONC}$ ) are both influenced by the older age of the 

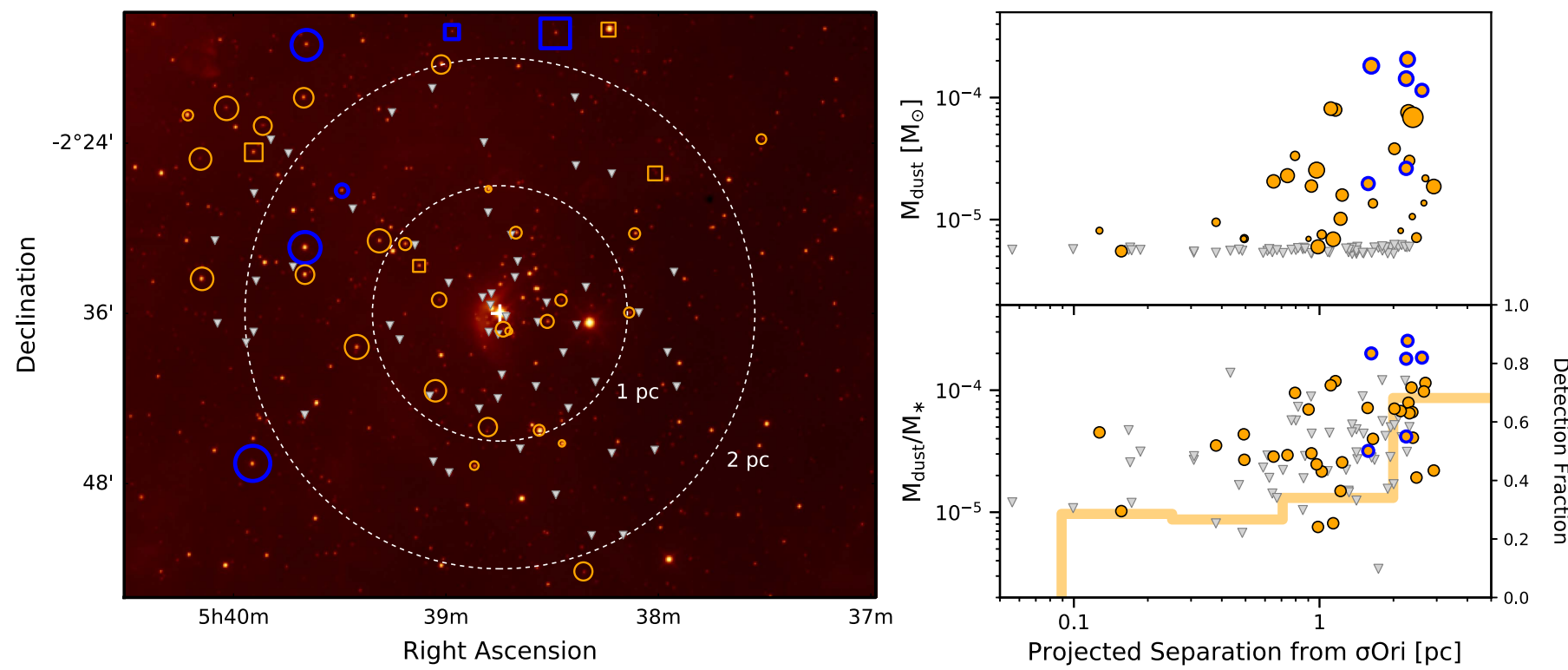

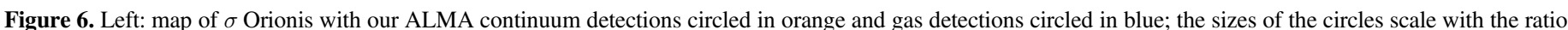

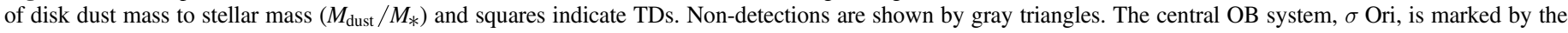

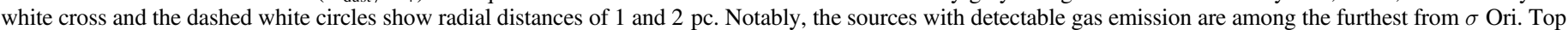

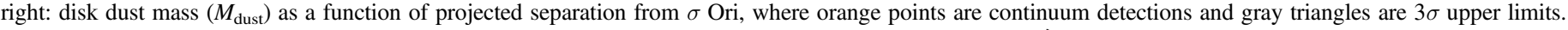

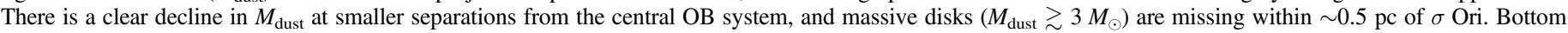

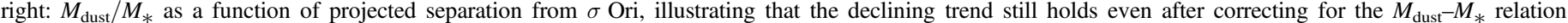
(Section 6.2). Our ALMA continuum detection fraction, shown by the thick orange line, also stays relatively constant until $\sim 2$ pc, after which it doubles.

region. Namely, dust mass distributions are known to decline with cluster age (Section 6.3) and typical intra-cluster velocity dispersions of several $\mathrm{km} \mathrm{s}^{-1}$ can result in cluster expansions of several parsecs by the age of $\sigma$ Orionis.

One concern is that, due to the $M_{\mathrm{dust}}-M_{*}$ relation (Section 6.2), mass segregation in clusters could produce these observed trends, if the least massive stars are preferentially located closer to the cluster centers. Mann et al. (2014) could not test this in the ONC because the nature of proplyds complicates any estimates of stellar mass. Because we can estimate stellar masses in $\sigma$ Orionis (Section 2), we also show in Figure 6 (lower right panel) the ratio of disk dust mass to stellar mass $\left(M_{\mathrm{dust}} / M_{*}\right)$ as a function of projected separation, confirming that the distance-dependent trend still holds even when accounting for stellar mass differences. Moreover, Figure 6 shows our ALMA continuum detection fraction, illustrating a relatively constant detection rate of $\sim 30 \%$ out to $\sim 2 \mathrm{pc}$, after which the detection fraction more than doubles to $\sim 70 \%$. Hernández et al. (2007) did not find a similar change in detection fraction with their Spitzer survey of $\sigma$ Orionis disks (see their Figure 16); however, this may be because external photoevaporation does not remove the inner (and therefore more gravitationally bound) disk regions probed by Spitzer.

Interestingly, we also find that the $\mathrm{CO}$ detections in our sample (blue circles in Figure 6) only exist in the outer regions of the cluster. This is qualitatively consistent with the picture of external photoevaporation: for typical disks, the gas is generally more extended than the dust, and therefore less tightly bound to the star, making the gas more susceptible to external photoevaporation. However, our gas sample is small and Mann et al. (2014) were unable to reliably detect gas in ONC disks due to cloud confusion, making it important to confirm our finding with surveys of other $\mathrm{OB}$ associations. If external photoevaporation does have a more significant effect on gas relative to dust, this would impact the types of planets that can form in OB associations.

Some evidence for external photoevaporation has been previously found for $\sigma$ Orionis disks. Rigliaco et al. (2009) detected strong optical forbidden emission lines from SO 587, which they interpreted as an externally driven photoevaporative flow due to the very low stellar mass accretion rate for this source, the profile shapes and luminosities of the forbidden emission lines, and the small projected separation $(\sim 0.3 \mathrm{pc})$ of the disk from $\sigma$ Ori (we did not detect this source with our ALMA observations). Additionally, Maucó et al. (2016) fit irradiated accretion disk models to the SEDs of 18 sources in $\sigma$ Orionis to show decreased disk masses and sizes when compared to those in the younger ONC. They interpreted this as evidence for external photoevaporation; however, their results were uncertain due to various model assumptions (e.g., constant $\alpha=0.01$ ) as well as the comparison of disk properties derived from disparate methods (e.g., they compared $\sigma$ Orionis disk radii derived from SED modeling, which probes the dust disk, to ONC disk radii derived from Hubble imagery, which probes the gas disk). Our ALMA observations therefore provide the clearest evidence to date that external photoevaporation is affecting disk masses throughout the $\sigma$ Orionis region.

Our findings also indicate that FUV (not just EUV) emission from OB stars is an important driver of external photoevaporation. Assuming a typical O9V FUV luminosity of $\log \left(L_{\mathrm{FUV}} / L_{\odot}\right)=$ 4.5 for $\sigma$ Ori, the geometrically diluted FUV flux within the region can be expressed as $\sim 8000(d / \mathrm{pc})^{-2} G_{0}$, where $d$ represents the distance from the photoevaporative source in parsecs and $G_{0}=1.6 \times 10^{-3} \mathrm{erg} \mathrm{cm}^{-2} \mathrm{~s}^{-1}$ (Habing 1968). We note that, although $\sigma$ Ori is a triple system, the FUV flux is usually dominated by the most massive star in the cluster (Fatuzzo \& Adams 2008; Holden et al. 2011). In this simple calculation, we also do not consider any extinction due to intra-cluster dust, 
which is observed to be at low densities in $\sigma$ Orionis (Walter et al. 2008), unlike in the ONC and NGC 2024. Figure 6 shows that external photoevaporation is affecting disk masses out to at least $\sim 2 \mathrm{pc}$, which when combined with the above equation corresponds to FUV fluxes $\gtrsim 2000 G_{0}$.

This supports recent observations that suggest even moderate FUV fluxes can drive significant mass loss. Kim et al. (2016) observed seven proplyds near a B star in NGC 1997, finding high mass-loss rates for an FUV flux of only $\sim 3000 G_{0}$. Haworth et al. (2017) also showed that the outer disk of IM Lup may be undergoing photoevaporation from an FUV flux of just $\sim 4 G_{0}$, where the high mass-loss rate can be explained by the large size of the disk (Cleeves et al. 2016), which causes gas in the outer regions to be only weakly gravitationally bound to the central star. Futhermore, Guarcello et al. (2016) found that disk frequency (as probed by near-IR excess) declines with smaller projected separation from the $\mathrm{OB}$ stars in Cygnus OB2 for FUV fluxes $\gtrsim 1000 G_{0}$, similar to what we calculated for $\sigma$ Orionis.

Together, these observations support recent theoretical findings by Facchini et al. (2016) and Haworth et al. (2016), who predicted high mass-loss rates due to external photoevaporation for FUV fluxes $<3000 G_{0}$. Moreover, these slow photoevaporative winds should be much more effective at removing gas and small $(\lesssim 1 \mu \mathrm{m})$ dust particles compared to larger ( $\gtrsim 1 \mathrm{~mm}$ ) solids (Facchini et al. 2016), which may help to explain our lack of gas detections at projected distances of $\lesssim 1.5 \mathrm{pc}$ from $\sigma$ Ori.

Finally, we showed in Section 5.3 that the average dust mass of the undetected sources in $\sigma$ Orionis is at least $\sim 5 \times$ lower than the smallest dust mass among the continuum detections, implying that disk dispersal occurs on short timescales. The rapid dispersal of disks impinged by intermediate external FUV fluxes has been predicted by Clarke (2007) and later by Anderson et al. (2013). Their models combined estimated mass-loss rates from external FUV photoevaporation with viscous disk evolution to show that disks should be dispersed from the outside-in on timescales much shorter than the expected disk lifetime. The typical lifetime of a viscous disk impinged with a $\sim 3000 G_{0}$ FUV flux was predicted to be roughly a few Myr, in agreement with our observations.

In summary, our observations indicate that external photoevaporation due to FUV emission from OB stars is significantly affecting disk evolution throughout the $\sigma$ Orionis cluster, though other disk evolution mechanisms are also clearly at play (Sections 6.2 and 6.3). This additional depletion of dust and gas for disks in OB clusters should have implications for planet formation, and detailed theoretical studies will be needed to quantify the impacts for different planet types and to identify any relations to trends seen in the exoplanet population.

\section{2. $M_{\text {dust }}-M_{*}$ Relation}

A clear correlation seen across protoplanetary disk populations is the positive relation between $M_{\text {dust }}$ and $M_{*}$ (e.g., Figure 7). Evidence for the $M_{\text {dust }}-M_{*}$ relation was first identified in pre-ALMA surveys of the Taurus star-forming region (Natta et al. 2000; Andrews et al. 2013) then later recovered with ALMA for other star-forming regions including the similarly aged Lupus clouds (Ansdell et al. 2016) and Chamaeleon I region (Pascucci et al. 2016) as well as the more evolved Upper Sco association (Barenfeld et al. 2016).
The $M_{\text {dust }}-M_{*}$ relation is pertinent to understanding planet formation because it tells us how disks are related to the properties of their host stars, which can then be tied to similar trends seen in the exoplanet population. For example, as previously noted by Andrews et al. (2013), the $M_{\text {dust }}-M_{*}$ relation may fundamentally explain the correlation between giant planet frequency and host star mass (Endl et al. 2006; Johnson et al. 2007; Bowler et al. 2010; Bonfils et al. 2013), as the cores of giant planets theoretically form more efficiently both in higher-mass disks (e.g., Thommes et al. 2008; Mordasini et al. 2012) and around higher-mass stars (e.g., Kennedy \& Kenyon 2008). Moreover, tracking the evolution of the $M_{\text {dust }}-M_{*}$ relation with age can tell us whether disk evolution proceeds differently around low-mass stars compared to high-mass stars, which in turn can help to constrain disk evolution theory (e.g., Pascucci et al. 2016).

However, parameterizing the $M_{\text {dust }}-M_{*}$ relation is complicated by three main factors: measurement uncertainties on both variables, intrinsic scatter in the data, and upper limits. The procedure most often utilized in the disk survey literature is the Bayesian linear regression method of Kelly (2007), as it is capable of accounting for these three key factors simultaneously, unlike other linear regression methods (see Pascucci et al. 2016 for a detailed discussion). For a given data set, the Kelly (2007) procedure fits a slope $(\beta)$, intercept $(\alpha)$, and intrinsic dispersion $(\delta)$ with associated uncertainties.

Using this method, Ansdell et al. (2016) showed that the fitted slopes to the protoplanetary disk populations in the young ( $1-3$ Myr) Taurus and Lupus regions were consistent with each other, and both shallower than that of the older ( 5-10 Myr) Upper Sco association. Pascucci et al. (2016) then showed that Chamaeleon I had a slope consistent with the similarly aged Taurus and Lupus regions, further supporting a steepening of the $M_{\text {dust }}-M_{*}$ relation with age. They also compared their results to theoretical models of grain growth, drift, and fragmentation to show that a steepening of the $M_{\text {dust }}{ }^{-}$ $M_{*}$ relation with age is consistent with the outer disk being in the fragmentation-limited regime. In this regime, grain sizes in the outer disk are limited by fragmenting collisions. When fragmentation sets the largest grain size, inward radial drift of dust occurs more rapidly around lower-mass stars, making their (sub-)millimeter continuum emission weaker and more compact with age compared to higher-mass stars.

Here we derive the $M_{\text {dust }}-M_{*}$ relation for $\sigma$ Orionis disks, again using the Bayesian linear regression method of Kelly (2007). We only consider sources in our ALMA sample with $M_{*} \geqslant 0.1 M_{\odot}$, so that we can compare our results to the relations derived for other star-forming regions (see below; this only removes five sources from our sample and does not affect the fit results). Using the $M_{*}$ and $M_{\text {dust }}$ values in Table 1, we derive a linear fit with $\alpha=1.0 \pm 0.2, \beta=2.0 \pm 0.4$, and $\delta=0.6 \pm 0.1 \mathrm{dex}$, as shown in Figure 7. To help illustrate that the fit is properly accounting for the numerous non-detections, we also show $3 \sigma$ upper limits from stacks of the non-detections in several stellar mass bins.

To compare our $M_{\text {dust }}-M_{*}$ relation derived for $\sigma$ Orionis to those found for other star-forming regions in a consistent manner, we follow the procedure described in Ansdell et al. (2016). Namely, we calculate $M_{\text {dust }}$ uniformly across each region by inputting the (sub-)millimeter continuum fluxes (or $3 \sigma$ upper limits) from the literature into Equation (1), along with the cluster distances and observation wavelengths of the 


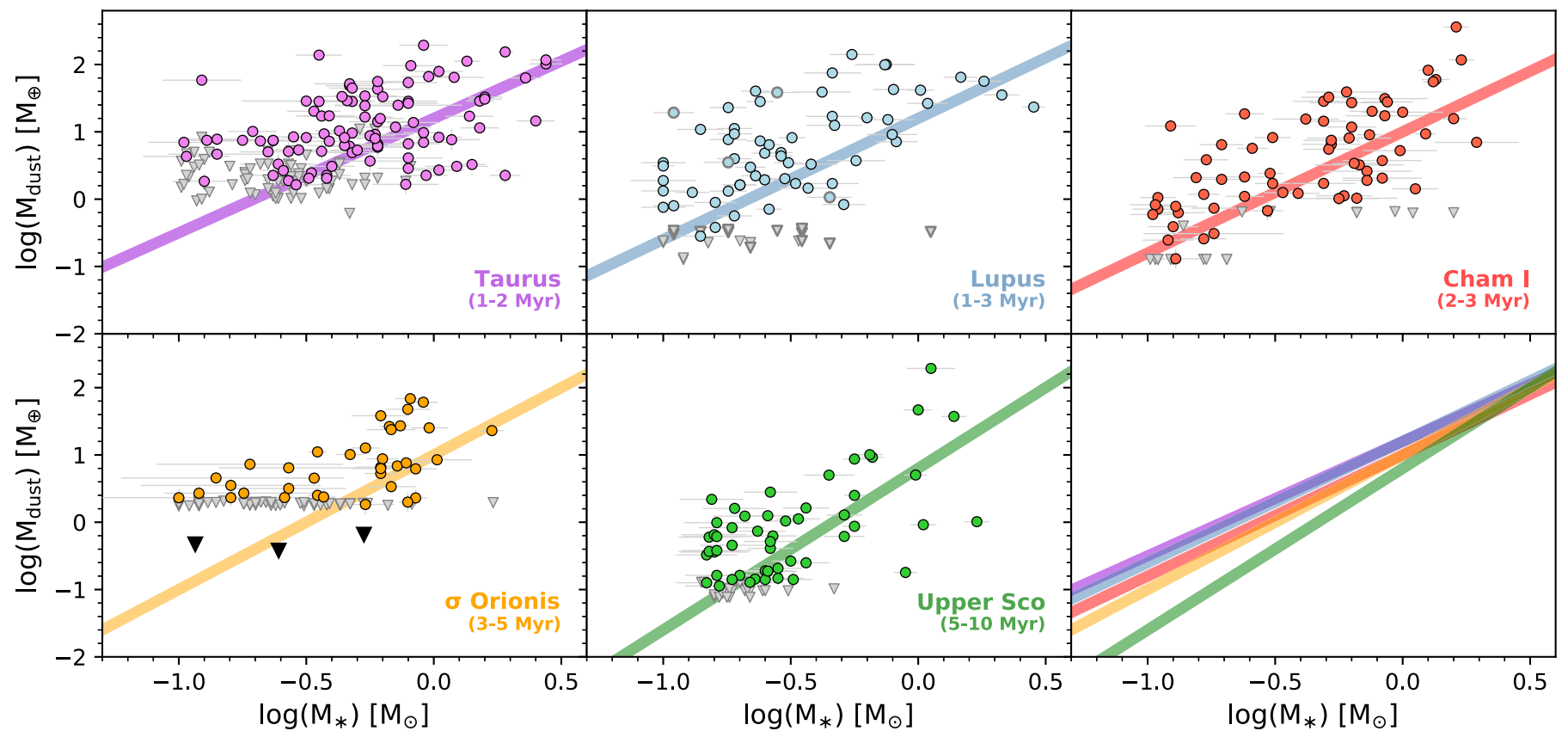

Figure 7. Disk dust mass $\left(M_{\text {dust }}\right)$ as a function of stellar mass $\left(M_{*}\right)$ for disk populations in five star-forming regions with ages spanning the disk dispersal timescale ( 1-10 Myr). Colored circles are (sub-)millimeter continuum detections and gray triangles are $3 \sigma$ upper limits. For $\sigma$ Orionis, the black triangles indicate $3 \sigma$ upper limits from stacks of the non-detections in three stellar mass bins. For Lupus, the 20 sources with unknown stellar masses that were included in the analysis via an MC method (see Ansdell et al. 2016) are given representative values and identified by thick gray outlines. For each region, the solid lines show the Bayesian linear regression fits to the data, which take into account upper limits, intrinsic scatter, and measurement errors on both axes (Kelly 2007). The lower right panel compares the fits in all five regions, illustrating the $\sim 1$ dex difference in $M_{\text {dust }}$ between the youngest and oldest regions at low stellar masses, and the convergence in $M_{\text {dust }}$ at high stellar masses.

surveys. We assume $T_{\text {dust }}=20 \mathrm{~K}$ for all disks and adopt distances of $140 \mathrm{pc}$ for Taurus (Kenyon et al. 2008), $150 \mathrm{pc}$ or 200 pc for Lupus (Comerón 2008), 160 pc for Chamaeleon I (Luhman 2008), and 145 pc for Upper Sco (de Zeeuw et al. 1999). For Upper Sco, we only include the "full," "evolved," and "transitional" disks from the sample of Barenfeld et al. (2016), as these represent the "primordial" disks that do not yet show signs of disk clearing. Only sources with $M_{*} \geqslant 0.1 M_{\odot}$ were considered in order to exclude brown dwarfs, while also maintaining a common stellar mass limit among the surveys. Stellar masses taken from the literature were derived using the Siess et al. (2000) evolutionary tracks for all regions except Chamaeleon I, which used the Baraffe et al. (2015) models; the stellar masses derived using these two grids are generally consistent, thus any effects should be negligible.

The fitted linear regression parameters for each region are given in Table 4 and plotted in Figure 7. Our results further support the steepening of the $M_{\text {dust }}-M_{*}$ relation with age, though the errors are large. Interestingly, we also find similarly large intrinsic dispersions for all five regions; as previously noted by Pascucci et al. (2016), this seems to be an inherent property of disk populations, reflecting a range of disk conditions (e.g., dust opacities, disk evolutionary states, and dust temperatures) rather than the age and/or environment of the region, and may partially account for the diversity seen in the exoplanet population.

We note that our fitted values in Table 4 are mostly consistent with those found by Pascucci et al. (2016; see their Table 4) despite differences in assumptions of grain opacity (e.g., they use $\beta=0.4$ in Equation (1), while we use $\beta=1.0$ ) and stellar mass cutoffs (e.g., they include sources with $M_{*}<0.1 M_{\odot}$, while we exclude brown dwarfs). Indeed, the
Table 4

$M_{\text {dust }}-M_{*}$ Bayesian Fit Parameters

\begin{tabular}{lcccc}
\hline \hline Region & Age $(\mathrm{Myr})$ & $\alpha^{\mathrm{b}}$ & $\beta^{\mathrm{b}}$ & $\delta$ \\
\hline Taurus & $1-2$ & $1.2 \pm 0.1$ & $1.7 \pm 0.2$ & $0.7 \pm 0.1$ \\
Lupus $^{\mathrm{a}}$ & $1-3$ & $1.2 \pm 0.2$ & $1.8 \pm 0.4$ & $0.9 \pm 0.1$ \\
Cha I & $2-3$ & $1.0 \pm 0.1$ & $1.8 \pm 0.3$ & $0.8 \pm 0.1$ \\
$\sigma$ Orionis & $3-5$ & $1.0 \pm 0.2$ & $2.0 \pm 0.4$ & $0.6 \pm 0.1$ \\
Upper Sco & $5-11$ & $0.8 \pm 0.2$ & $2.4 \pm 0.4$ & $0.7 \pm 0.1$ \\
\hline
\end{tabular}

Notes.

${ }^{\text {a }}$ Fit taken from Ansdell et al. (2016), as they used the same methodology described in Section 6.2, but also an MC analysis to account for 20 Lupus sources with unknown stellar masses.

${ }^{\mathrm{b}}$ We use the convention of Kelly (2007), where $\beta$ and $\alpha$ represent the slope and intercept, respectively. This differs from that of Pascucci et al. (2016), who switched these symbols.

main disagreement is the intercept estimate for Lupus, which differs because Pascucci et al. (2016) exclude the 20 sources in Lupus with unknown stellar masses, while we account for them using the MC approach described in Ansdell et al. (2016). The slope for Upper Sco is also noticeably different (although within errors) because Pascucci et al. (2016) only consider "full" and "transitional" disks from Upper Sco, while we also include "evolved" disks following the definition of "primordial" disks in Barenfeld et al. (2016).

Finally, we address three potential caveats to our Bayesian linear regression fit to $\sigma$ Orionis disks. First, Pascucci et al. (2016) found that shallower slopes can result when the sample is dominated by upper limits at low stellar masses. Although roughly two-thirds of our $\sigma$ Orionis sample was undetected in the continuum, the non-detections span a range of stellar masses (see Figure 7); moreover, even if the slope is actually 
steeper, this would only further distinguish $\sigma$ Orionis from the younger regions. Second, the source with the highest stellar mass in $\sigma$ Orionis is not detected in the continuum, which could potentially skew the fit. We could not find a valid reason for discarding this source (e.g., no evidence for binarity), though its spectral type is uncertain $( \pm 2.5$ spectral types; Hernández et al. 2014). Nevertheless, when re-fitting the distribution without this source, we recover the same parameters. Third, we have shown in Section 6.1 that external photoevaporation is reducing disk dust masses in $\sigma$ Orionis. This may serve to steepen the $M_{\text {dust }}-M_{*}$ relation, as external photoevaporation should be more effective at removing gas and small dust grains around lower-mass stars whose orbiting material is less gravitationally bound. Unfortunately, this possible effect has not yet been tested with external photoevaporation models. Given these potential issues, a more sensitive (sub-)millimeter continuum survey of $\sigma$ Orionis, as well as additional observations and detailed modeling, will be needed to confirm the linear regression fit presented here. Furthermore, we note that several other linear regression methods for left-censored data sets are available in the statistical literature and should be tested for this specific astronomical problem.

\subsection{Dust-mass Distributions}

Disk dispersal and grain growth should be reflected in a steady decline with age of the bulk dust mass probed by (sub-)millimeter continuum flux. Ansdell et al. (2016) showed that the average dust mass of disks in a given star-forming region, as measured from (sub-)millimeter continuum flux, does indeed decline with age. Specifically, they found that Lupus and Taurus have consistent mean dust masses $\left(15 \pm 3 M_{\oplus}\right.$ and $15 \pm 2 M_{\oplus}$, respectively), while Upper Sco has a mean dust mass that is $\sim 5 \times$ lower $\left(5 \pm 3 M_{\oplus}\right)$. They calculated dust masses uniformly across each region (as described in Section 6.2) then derived the average dust mass using the Kaplan-Meier estimator in the ASURV package (Lavalley et al. 1992) to properly account for the upper limits using well-established techniques for left-censored data sets. We follow this procedure to now include results from our ALMA survey of $\sigma$ Orionis disks as well as the ALMA survey of Chamaeleon I disks (Pascucci et al. 2016). As shown in Figure 8, we find average disk dust masses of $7 \pm 1 M_{\oplus}$ for $\sigma$ Orionis and $13 \pm 4 M_{\oplus}$ for Chamaeleon I, further supporting the decline of average disk dust mass with age.

When comparing the dust-mass distributions of two regions, it is important to confirm that they have similar stellar mass distributions due to the $M_{\text {dust }}-M_{*}$ relation discussed in Section 6.2. We therefore employ the two-sample tests in the ASURV package to determine the probabilities that the stellar masses of each region are drawn from the same parent population. We find that the stellar mass distributions are statistically indistinguishable for $\sigma$ Orionis and Lupus ( $p=$ $0.45-0.96), \sigma$ Orionis and Chamaeleon I $(p=0.06-0.22)$, and $\sigma$ Orionis and Upper Sco $(p=0.21-0.30)$. However, we found statistically distinct stellar mass distributions for $\sigma$ Orionis and Taurus $(p=0.03-0.04)$. Therefore, we can directly compare the dust-mass distribution of $\sigma$ Orionis to those of Lupus, Chamaeleon I, and Upper Sco in Figure 8. However, caution should be taken when interpreting the comparison of $\sigma$ Orionis to Taurus in Figure 8 due to their potentially different stellar mass distributions. Nevertheless, our main conclusions in the previous paragraph remain the same.

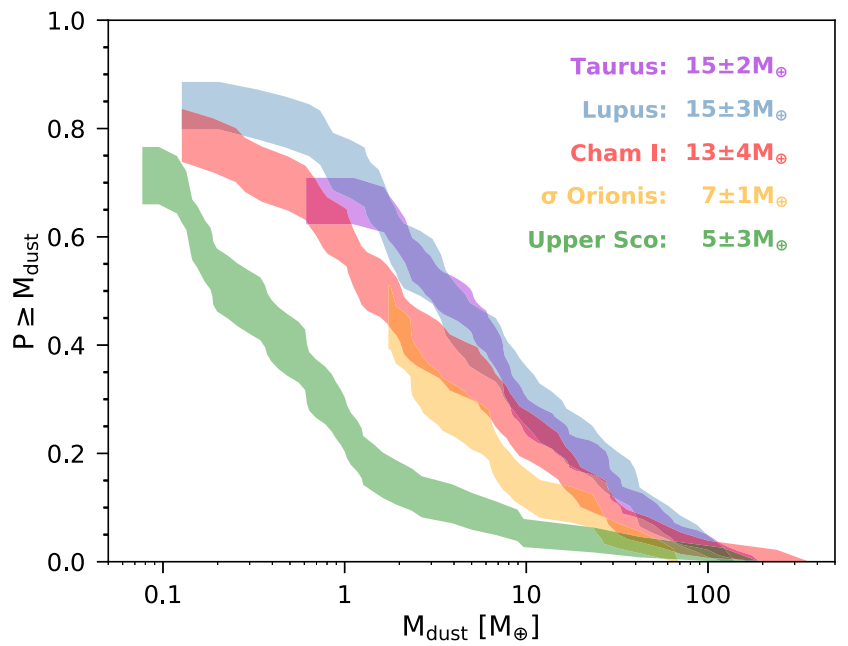

Figure 8. Dust-mass cumulative distributions for Taurus, Lupus, Chamaeleon I, $\sigma$ Orionis, and Upper Sco disks around host stars with $M_{*} \geqslant 0.1 M_{\odot}$ The average dust mass for each region is given for reference. The distributions and their $1 \sigma$ confidence intervals were calculated using the Kaplan-Meier estimator in the ASURV package (Lavalley et al. 1992) to properly account for upper limits using well-established techniques for left-censored data sets.

Alternatively, to account for stellar mass differences between regions, one could instead compare the $M_{\text {dust }} / M_{*}$ distributions (e.g., Barenfeld et al. 2016) or employ an MC approach that aims to normalize the stellar mass selection functions (e.g., Andrews et al. 2013; Williams et al. 2013; Ansdell et al. 2016). However, in this work, we do not attempt these more detailed analyses given the larger uncertainties on our $M_{*}$ estimates, especially for the sources with photometrically derived stellar masses.

\subsection{Ingredients for Giant Planet Formation}

Core accretion theory predicts that giant planets form when solid cores of a minimum critical mass assemble in the disk, enabling runaway accretion of the surrounding gaseous material (Pollack et al. 1996; Ida \& Lin 2004). The accretion of a gaseous envelope is expected to occur rapidly, where $\sim 10 M_{\oplus}$ cores reach masses of $\sim 1 M_{\text {Jup }}$ within $\sim 0.1$ Myr. Within the framework of this model, we can constrain the occurrence of giant planet formation by observing how quickly the dust and gas content in typical protoplanetary disks depletes to levels below what are thought to be needed to form a gas giant.

In particular, we can look at the fraction of protoplanetary disks in a region with dust masses above the $\sim 10 M_{\oplus}$ limit needed to form a giant planet core. For regions at $\sim 1-3 \mathrm{Myr}$, we see roughly a quarter of protoplanetary disks above this threshold (30\% in Taurus, 26\% in Lupus, and 23\% in Chamaeleon I). At $\sim 3-5 \mathrm{Myr}$, we see this fraction cut in half (13\% in $\sigma$ Orionis) and then halved again at $\sim 5-10 \mathrm{Myr}(5 \%$ in Upper Sco). Although these are only rough estimates due to different survey completenesses, they seem to clearly reflect a sharp decline in the capacity of disks to form giant planets with age. Even in the youngest regions, the majority of disks appear to lack sufficient dust to form the solid cores needed to build giant planets, implying that giant planet formation is either rare or well on its way after just a few Myr.

Additionally, stacking (sub-)millimeter continuum nondetections allows us to put limits on the average amount of 
dust in the lowest-mass disks (e.g., Section 5.3). In Lupus, Ansdell et al. (2016) found that the undetected disks had extremely low average dust masses of $\lesssim 6$ Lunar masses $\left(0.03 M_{\oplus}\right)$, comparable to debris disk levels (Wyatt 2008). Although the further distance of $\sigma$ Orionis results in looser constraints, we still find that undetected disks have $\lesssim 4$ Martian masses $\left(0.4 M_{\oplus}\right)$ of dust on average (Section 5.3). These findings support theoretical predictions that viscous disks evolve rapidly into debris disks once stellar accretion ceases and photoevaporation from the central star dominates, clearing the dust from the inside out and leaving behind larger solids such as pebbles and planetesimals (e.g., Clarke et al. 2001; Alexander et al. 2006). Previous studies of weak-lined T Tauri stars have provided observational evidence for rapid disk clearing (e.g., Cieza et al. 2013; Hardy et al. 2015), and Panić et al. (2013) used a compilation of sub-millimeter fluxes from the literature to show that debris disks are substantially less massive than disks around younger pre-main-sequence stars. However, our larger and more homogeneous samples of Lupus and $\sigma$ Orionis disks confirm that rapid disk clearing is a uniform occurrence, even among young protoplanetary disk populations.

Another ingredient for giant planet formation is of course the gas. Although bulk gas masses are notoriously difficult to measure (Section 5.2), the fact that we found only three disks in $\sigma$ Orionis that exhibit both ${ }^{12} \mathrm{CO}$ and ${ }^{13} \mathrm{CO}$ emission is telling; indeed, their line fluxes correspond to gas masses of just $\sim 2-7 M_{\text {Jup }}$ using the methodology described in Section 5.2. For the remaining disks in $\sigma$ Orionis, we find upper limits on their individual gas masses of just $\sim 3 M_{\text {Jup }}$. Moreover, the average gas mass of disks detected in the continuum but undetected in ${ }^{12} \mathrm{CO}$ is $<1.0 \mathrm{M}_{\text {Jup }}$ (Section 5.3). These low gas masses again suggest that giant planet formation is either rare or nearly complete by the $\sim 3-5 \mathrm{Myr}$ age of $\sigma$ Orionis (or that carbon is being significantly depleted in protoplanetary disks; see Section 5.2).

\subsection{Relation between $M_{\mathrm{disk}}$ and $\dot{M}_{\mathrm{acc}}$}

Viscously evolving protoplanetary disks should exhibit a direct relation between their total disk mass $\left(M_{\text {disk }}\right)$ and stellar mass accretion rate $\left(\dot{M}_{\text {acc }}\right)$, as shown in Hartmann et al. (1998). This theoretical prediction was only recently confirmed observationally by Manara et al. (2016), who combined the ALMA (Ansdell et al. 2016) and VLT/X-Shooter (Alcalá et al. 2014, 2016) surveys of protoplanetary disks in the Lupus clouds to reveal a linear relation between $\dot{M}_{\text {acc }}$ and $M_{\text {disk }}$. Manara et al. (2016) also showed that, when assuming an ISM gas-to-dust ratio of $\sim 100$, the $M_{\text {disk }} / \dot{M}_{\text {acc }}$ ratio is consistent with the age of the region, as expected for viscously evolving disks. These observational findings suggest that mass accretion onto the stellar surface is indeed related to the properties of the outer disk.

To search for a similar correlation in $\sigma$ Orionis, we combine our ALMA observations with the $U$-band survey of Rigliaco et al. (2011). The latter used their photometric data to estimate $\dot{M}_{\text {acc }}$, making these accretion rates more uncertain than those obtained with spectroscopy for Lupus disks. As shown in Figure 9, only 20 sources (the most massive disks in $\sigma$ Orionis) have both $M_{\text {dust }}$ estimates and constraints on $\dot{M}_{\text {acc }}$ (the latter of which are mostly upper or lower limits). The sparseness of this sub-sample, combined with the smaller $M_{\text {dust }}$ range when compared to the Lupus sample, limits our ability to fit a relation

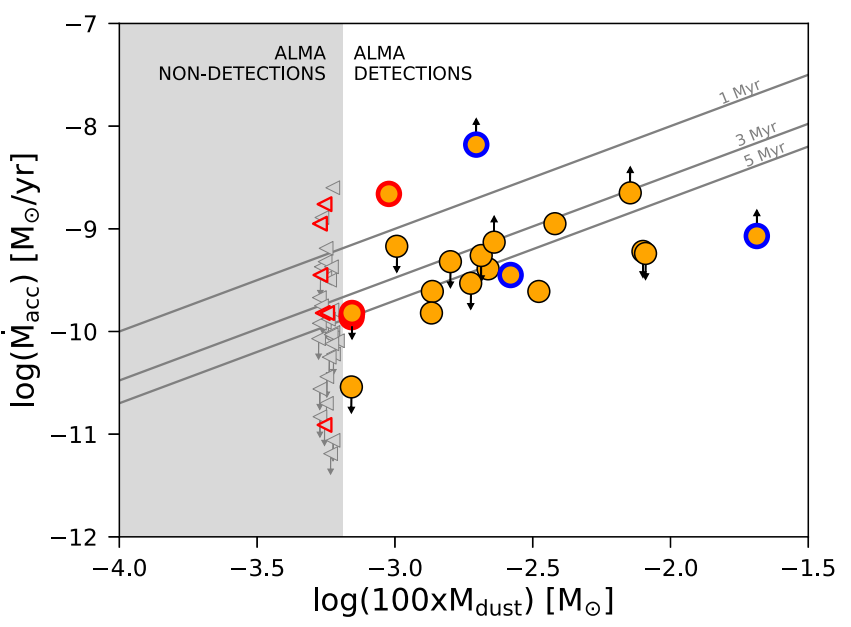

Figure 9. Stellar mass accretion rate $\left(\dot{M}_{\text {acc }}\right)$ from the $U$-band survey of Rigliaco et al. (2011) vs. disk mass $\left(M_{\text {disk }}=100 \times M_{\text {dust }}\right)$ from our ALMA observations, for the $\sigma$ Orionis members included in both surveys. Orange circles are ALMA continuum detections and gray triangles are $3 \sigma$ upper limits. Upward/downward arrows are lower/upper limits on $\dot{M}_{\text {acc }}$. Sources outlined in blue are our CO detections (Section 4.2) and sources outlined in red are $\lesssim 0.5 \mathrm{pc}$ from the central OB system (Section 6.1). The diagonal lines show different $M_{\text {disk }} / \dot{M}_{\text {acc }}$ ratios, which represents different viscous timescales.

similar to that in Manara et al. (2016). However, we note that, as for Lupus disks, the $M_{\text {disk }} / \dot{M}_{\text {acc }}$ ratios are consistent with the age of $\sigma$ Orionis for an ISM gas-to-dust ratio of $\sim 100$.

The disks undetected with ALMA (i.e., the least massive disks in $\sigma$ Orionis) span over 2 dex in $\dot{M}_{\text {acc }}$, and thus a correspondingly large range of $M_{\text {disk }} / \dot{M}_{\text {acc }}$ ratios, as shown in Figure 9. The undetected disks with low $\dot{M}_{\text {acc }}$ values or upper limits are consistent with expectations from viscous evolution. However, the undetected disks with moderate-to-high $\dot{M}_{\text {acc }}$ values $\left(\gtrsim 2 \times 10^{-10} M_{\odot} \mathrm{yr}^{-1}\right)$ are unexpected, as these should have lifetimes much shorter than the age of the region. The sources $\lesssim 0.5 \mathrm{pc}$ from the central OB star (outlined in red in Figure 9) are most readily explained by external photoevaporation (see Section 6.1), which would serve to reduce $M_{\text {disk }}$ by removing mass from the outer disk, thereby increasing the $M_{\text {disk }} / \dot{M}_{\text {acc }}$ ratio. Although this accounts for only a handful of objects, there is evidence that external photoevaporation is occurring throughout the region (Section 6.1), thus may apply to more of the undetected disks. Additionally, the $\dot{M}_{\text {acc }}$ values estimated from $U$-band photometry are uncertain and need to be confirmed with spectroscopy. Nevertheless, there are a sufficient number of sources with low disk masses and significant accretion rates to warrant further investigation. These objects might have strongly variable accretion rates, or alternatively the accreting gas may come from the evaporation of ice-coated dust grains.

\section{Summary}

We have used ALMA to conduct a high-sensitivity millimeter survey of protoplanetary disks in the $\sigma$ Orionis cluster. This region is particularly interesting for studying disk evolution as its intermediate age $(\sim 3-5 \mathrm{Myr})$ is comparable to the median disk lifetime, and therefore corresponds to a potentially important phase of disk evolution and planet formation.

1. We used ALMA to survey the dust and gas in 92 protoplanetary disks around $\sigma$ Orionis members with 
$M_{*} \gtrsim 0.1 M_{\odot}$. Our observations cover the $1.33 \mathrm{~mm}$ continuum as well as the ${ }^{12} \mathrm{CO},{ }^{13} \mathrm{CO}$, and $\mathrm{C}^{18} \mathrm{O}$ $J=2-1$ lines. Out of the 92 sources, we detected only 37 in the millimeter continuum and 6 in ${ }^{12} \mathrm{CO}, 3$ in ${ }^{13} \mathrm{CO}$, and none in $\mathrm{C}^{18} \mathrm{O}$.

2. The continuum emission constrained dust masses to $\sim 2 M_{\oplus}$, while the CO line emission constrained gas masses to $\sim 3 M_{\text {Jup }}$. Only 11 disks had $M_{\text {dust }} \gtrsim 10 M_{\oplus}$, indicating that after a few Myr of evolution the vast majority of disks lack sufficient dust to form giant planet cores. The low gas masses also indicate that giant planet formation must be rapid or rare, but may also reflect significant carbon depletion in protoplanetary disks. Moreover, stacking the individually undetected continuum sources limited their average dust mass to $\sim 5 \times$ lower than that of the faintest detected disk, supporting theoretical models that predict disks dissipating rapidly once accretion stops and photoevaporation dominates.

3. We found that external photoevaporation from the central OB stars is influencing disk evolution throughout the region. Namely, disk dust masses clearly decline with decreasing projected separation from the photoionizing source, and the handful of cluster members with detected $\mathrm{CO}$ emission exist only at projected separations $>1.5 \mathrm{pc}$. This indicates that even moderate external FUV fluxes can result in significant mass-loss rates, and future theoretical studies will be needed to quantify the implications for planet formation in OB clusters.

4. Comparing the protoplanetary disk population in $\sigma$ Orionis to those of other star-forming regions provided continuing support of the steady decline in average disk dust mass and steepening of the $M_{\text {dust }}-M_{*}$ relation with age. Quantifying these evolutionary trends can help to determine the relative importance of different disk processes during key eras of planet formation. However, for $\sigma$ Orionis, these trends may also be influenced by the effects of external photoevaporation from the central $\mathrm{OB}$ stars.

5. Collectively, our findings indicate that giant planet formation is inherently rare and/or well underway by a few Myr of age. However, due to the abundance of upper limits in our ALMA sample, and the need for better constraints on stellar properties, a higher-sensitivity (sub-)millimeter survey as well as a complete spectroscopic survey of the members of $\sigma$ Orionis analyzed in this work should be conducted to confirm these results.

M.C.A. and J.P.W. were supported by NSF and NASA grants AST-1208911 and NNX15AC92G, respectively. M.C.A. acknowledges student observing support from NRAO. C.F.M. acknowledges an ESA Research Fellowship. N.M. is supported in part by the Beatrice W. Parrent Fellowship in Astronomy at the University of Hawaii. Leiden is supported by the European Union A-ERC grant 291141 CHEMPLAN, by the Netherlands Research School for Astronomy (NOVA), and by grant 614.001.352 from the Netherlands Organization for Scientific Research (NWO). This paper makes use of the following ALMA data: ADS/JAO.ALMA2015.1.00089.S. ALMA is a partnership of ESO (representing its member states), NSF (USA) and NINS (Japan), together with NRC (Canada), NSC and ASIAA (Taiwan), and KASI (Re- public of Korea), in cooperation with the Republic of Chile. The Joint ALMA Observatory is operated by ESO, AUI/NRAO and NAOJ. The National Radio Astronomy Observatory is a facility of the National Science Foundation operated under cooperative agreement by Associated Universities, Inc.

\section{References}

Adams, F. C., Hollenbach, D., Laughlin, G., \& Gorti, U. 2004, ApJ, 611, 360 Aikawa, Y., Umebayashi, T., Nakano, T., \& Miyama, S. M. 1997, ApJL, 486, L51

Alcalá, J. M., Manara, C. F., Natta, A., et al. 2017, A\&A, 600, A20

Alcalá, J. M., Natta, A., Manara, C. F., et al. 2014, A\&A, 561, A2

Alexander, R., Pascucci, I., Andrews, S., Armitage, P., \& Cieza, L. 2014, in Protostars and Planets VI, ed. H. Beuther et al. (Tucson, AZ: Univ. Arizona Press), 475

Alexander, R. D., Clarke, C. J., \& Pringle, J. E. 2006, MNRAS, 369, 229

Anderson, K. R., Adams, F. C., \& Calvet, N. 2013, ApJ, 774, 9

Andrews, S. M., Rosenfeld, K. A., Kraus, A. L., \& Wilner, D. J. 2013, ApJ, 771,129

Andrews, S. M., \& Williams, J. P. 2005, ApJ, 631, 1134

Andrews, S. M., Wilner, D. J., Espaillat, C., et al. 2011, ApJ, 732, 42

Andrews, S. M., Wilner, D. J., Hughes, A. M., Qi, C., \& Dullemond, C. P. 2009, ApJ, 700, 1502

Ansdell, M., Williams, J. P., \& Cieza, L. A. 2015, ApJ, 806, 221

Ansdell, M., Williams, J. P., van der Marel, N., et al. 2016, ApJ, 828, 46

Baraffe, I., Homeier, D., Allard, F., \& Chabrier, G. 2015, A\&A, 577, A42

Barenfeld, S. A., Carpenter, J. M., Ricci, L., \& Isella, A. 2016, ApJ, 827, 142

Beckwith, S. V. W., Sargent, A. I., Chini, R. S., \& Guesten, R. 1990, AJ, 99, 924

Bergin, E. A., Cleeves, L. I., Crockett, N., \& Blake, G. A. 2014, FaDi, 168 arXiv: 1405.7394

Bergin, E. A., Cleeves, L. I., Gorti, U., et al. 2013, Natur, 493, 644

Bergin, E. A., Hogerheijde, M. R., Brinch, C., et al. 2010, A\&A, 521, L33

Bik, A., Lenorzer, A., Kaper, L., et al. 2003, A\&A, 404, 249

Bitsch, B., Johansen, A., Lambrechts, M., \& Morbidelli, A. 2015, A\&A, 575, A28

Bohlin, R. C., Savage, B. D., \& Drake, J. F. 1978, ApJ, 224, 132

Bonfils, X., Delfosse, X., Udry, S., et al. 2013, A\&A, 549, A109

Borucki, W. J., Koch, D., Basri, G., et al. 2010, Sci, 327, 977

Bowler, B. P., Johnson, J. A., Marcy, G. W., et al. 2010, ApJ, 709, 396

Bruderer, S., van Dishoeck, E. F., Doty, S. D., \& Herczeg, G. J. 2012, A\&A, 541, A91

Caballero, J. A. 2008, A\&A, 478, 667

Cieza, L. A., Olofsson, J., Harvey, P. M., et al. 2013, ApJ, 762, 100

Clarke, C. J. 2007, MNRAS, 376, 1350

Clarke, C. J., Gendrin, A., \& Sotomayor, M. 2001, MNRAS, 328, 485

Cleeves, L. I., Öberg, K. I., Wilner, D. J., et al. 2016, ApJ, 832, 110

Comerón, F. 2008, in Handbook of Star Forming Regions, Volume II: The Southern Sky Monograph Publications, Vol. 5, ed. B. Reipurth (San Francisco, CA: ASP), 295

de Zeeuw, P. T., Hoogerwerf, R., de Bruijne, J. H. J., Brown, A. G. A., \& Blaauw, A. 1999, AJ, 117, 354

Du, F., Bergin, E. A., \& Hogerheijde, M. R. 2015, ApJL, 807, L32

Eistrup, C., Walsh, C., \& van Dishoeck, E. F. 2016, A\&A, 595, A83

Endl, M., Cochran, W. D., Kürster, M., et al. 2006, ApJ, 649, 436

Espaillat, C., Muzerolle, J., Najita, J., et al. 2014, in Protostars and Planets VI, ed. H. Beuther et al. (Tucson, AZ: Univ. Arizona Press), 497

Facchini, S., Clarke, C. J., \& Bisbas, T. G. 2016, MNRAS, 457, 3593

Fatuzzo, M., \& Adams, F. C. 2008, ApJ, 675, 1361

Favre, C., Cleeves, L. I., Bergin, E. A., Qi, C., \& Blake, G. A. 2013, ApJL, 776, L38

France, K., Herczeg, G. J., McJunkin, M., \& Penton, S. V. 2014, ApJ, 794, 160

Frerking, M. A., Langer, W. D., \& Wilson, R. W. 1982, ApJ, 262, 590

Fressin, F., Torres, G., Charbonneau, D., et al. 2013, ApJ, 766, 81

Gaidos, E., Mann, A. W., Kraus, A. L., \& Ireland, M. 2016, MNRAS, 457, 2877

Guarcello, M. G., Drake, J. J., Wright, N. J., et al. 2016, arXiv:1605.01773

Guidi, G., Tazzari, M., Testi, L., et al. 2016, A\&A, 588, A112

Habing, H. J. 1968, BAN, 19, 421

Hardy, A., Caceres, C., Schreiber, M. R., et al. 2015, A\&A, 583, A66

Hartmann, L., Calvet, N., Gullbring, E., \& D’Alessio, P. 1998, ApJ, 495, 385

Haworth, T. J., Boubert, D., Facchini, S., Bisbas, T. G., \& Clarke, C. J. 2016, MNRAS, 463, 3616

Haworth, T. J., Facchini, S., Clarke, C. J., \& Cleeves, L. I. 2017, MNRAS, submitted 
Herczeg, G. J., \& Hillenbrand, L. A. 2015, ApJ, 808, 23

Hernández, J., Calvet, N., Perez, A., et al. 2014, ApJ, 794, 36

Hernández, J., Hartmann, L., Megeath, T., et al. 2007, ApJ, 662, 1067

Hildebrand, R. H. 1983, QJRAS, 24, 267

Hogerheijde, M. R., Bergin, E. A., Brinch, C., et al. 2011, Sci, 334, 338

Holden, L., Landis, E., Spitzig, J., \& Adams, F. C. 2011, PASP, 123, 14

Howard, A. W., Marcy, G. W., Bryson, S. T., et al. 2012, ApJS, 201, 15

Howard, A. W., Marcy, G. W., Johnson, J. A., et al. 2010, Sci, 330, 653

Ida, S., \& Lin, D. N. C. 2004, ApJ, 604, 388

Jeffries, R. D., Maxted, P. F. L., Oliveira, J. M., \& Naylor, T. 2006, MNRAS, 371, L6

Johnson, J. A., Butler, R. P., Marcy, G. W., et al. 2007, ApJ, 670, 833

Johnstone, D., Hollenbach, D., \& Bally, J. 1998, ApJ, 499, 758

Kama, M., Bruderer, S., Carney, M., et al. 2016, A\&A, 588, A108

Kelly, B. C. 2007, ApJ, 665, 1489

Kennedy, G. M., \& Kenyon, S. J. 2008, ApJ, 673, 502

Kenyon, S. J., Gómez, M., \& Whitney, B. A. 2008, in Handbook of Star Forming Regions, Volume I: The Northern Sky ASP Monograph Publications, Vol. 4, ed. B. Reipurth (San Francisco, CA: ASP), 405

Kim, J. S., Clarke, C. J., Fang, M., \& Facchini, S. 2016, ApJL, 826, L15

Lacy, J. H., Knacke, R., Geballe, T. R., \& Tokunaga, A. T. 1994, ApJL, 428, L69

Lavalley, M., Isobe, T., \& Feigelson, E. 1992, in ASP Conf. Ser. 25, Astronomical Data Analysis Software and Systems I, ed. D. M. Worrall, C. Biemesderfer, \& J. Barnes (San Francisco, CA: ASP), 245

Lee, E. J., \& Chiang, E. 2016, ApJ, 817, 90

Lee, N., Williams, J. P., \& Cieza, L. A. 2011, ApJ, 736, 135

Levine, J. L., Steinhauer, A., Elston, R. J., \& Lada, E. A. 2006, ApJ, 646, 1215

Luhman, K. L. 2008, in Handbook of Star Forming Regions, Volume II: The Southern Sky ASP Monograph Publications, Vol. 5, ed. B. Reipurth (San Francisco, CA: ASP), 169

Manara, C. F., Rosotti, G., Testi, L., et al. 2016, A\&A, 591, L3

Manara, C. F., Testi, L., Herczeg, G. J., et al. 2017, A\&A, in press (arXiv:1704. 02842)

Mann, R. K., Andrews, S. M., Eisner, J. A., et al. 2015, ApJ, 802, 77

Mann, R. K., Di Francesco, J., Johnstone, D., et al. 2014, ApJ, 784, 82

Maucó, K., Hernández, J., Calvet, N., et al. 2016, ApJ, 829, 38

Maxted, P. F. L., Jeffries, R. D., Oliveira, J. M., Naylor, T., \& Jackson, R. J. 2008, MNRAS, 385, 2210

Mayor, M., Marmier, M., Lovis, C., et al. 2011, arXiv:1109.2497

Miotello, A., van Dishoeck, E. F., Kama, M., \& Bruderer, S. 2016, A\&A, 594, A85

Miotello, A., van Dishoeck, E. F., Williams, J. P., et al. 2017, A\&A, 599, A113
Mordasini, C., Alibert, Y., Benz, W., Klahr, H., \& Henning, T. 2012, A\&A, 541, A97

Mordasini, C., van Boekel, R., Mollière, P., Henning, T., \& Benneke, B. 2016, ApJ, 832, 41

Natta, A., Grinin, V., \& Mannings, V. 2000, in Protostars and Planets IV, ed A. Boss, V. Mannings, \& S. S. Russell (Tucson, AZ: Univ. Arizona Press), 559

Oliveira, J. M., Jeffries, R. D., Kenyon, M. J., Thompson, S. A., \& Naylor, T. 2002, A\&A, 382, L22

Oliveira, J. M., Jeffries, R. D., \& van Loon, J. T. 2004, MNRAS, 347, 1327

Panić, O., Holland, W. S., Wyatt, M. C., et al. 2013, MNRAS, 435, 1037

Pascucci, I., Testi, L., Herczeg, G. J., et al. 2016, ApJ, 831, 125

Pecaut, M. J., Mamajek, E. E., \& Bubar, E. J. 2012, ApJ, 746, 154

Petigura, E. A., Marcy, G. W., \& Howard, A. W. 2013, ApJ, 770, 69

Pollack, J. B., Hubickyj, O., Bodenheimer, P., et al. 1996, Icar, 124, 62

Preibisch, T., Brown, A. G. A., Bridges, T., Guenther, E., \& Zinnecker, H. 2002, AJ, 124, 404

Rigliaco, E., Natta, A., Randich, S., \& Sacco, G. 2009, A\&A, 495, L13

Rigliaco, E., Natta, A., Randich, S., Testi, L., \& Biazzo, K. 2011, A\&A, 525, A47

Rigliaco, E., Natta, A., Testi, L., et al. 2012, A\&A, 548, A56

Ripple, F., Heyer, M. H., Gutermuth, R., Snell, R. L., \& Brunt, C. M. 2013, MNRAS, 431, 1296

Ros, K., \& Johansen, A. 2013, A\&A, 552, A137

Sacco, G. G., Franciosini, E., Randich, S., \& Pallavicini, R. 2008, A\&A, 488, 167

Schaefer, G. H., Hummel, C. A., Gies, D. R., et al. 2016, AJ, 152, 213

Schwarz, K. R., Bergin, E. A., Cleeves, L. I., et al. 2016, ApJ, 823, 91

Sherry, W. H., Walter, F. M., Wolk, S. J., \& Adams, N. R. 2008, AJ, 135, 1616

Shimajiri, Y., Kitamura, Y., Saito, M., et al. 2014, A\&A, 564, A68

Siess, L., Dufour, E., \& Forestini, M. 2000, A\&A, 358, 593

Störzer, H., \& Hollenbach, D. 1999, ApJ, 515, 669

Tazzari, M. 2017, A\&A, submitted

Thommes, E. W., Matsumura, S., \& Rasio, F. A. 2008, Sci, 321, 814

Walter, F. M., Sherry, W. H., Wolk, S. J., \& Adams, N. R. 2008, in Handbook of Star Forming Regions, Volume I: The Northern Sky ASP Monograph Publications, Vol. 4, ed. B. Reipurth (San Francisco, CA: ASP), 732

Weidenschilling, S. J. 1977, Ap\&SS, 51, 153

Williams, J. P. 2012, M\&PS, 47, 1915

Williams, J. P., \& Best, W. M. J. 2014, ApJ, 788, 59

Williams, J. P., \& Cieza, L. A. 2011, ARA\&A, 49, 67

Williams, J. P., Cieza, L. A., Andrews, S. M., et al. 2013, MNRAS, 435, 1671

Wyatt, M. C. 2008, ARA\&A, 46, 339

Yu, M., Willacy, K., Dodson-Robinson, S. E., Turner, N. J., \& Evans, N. J., II 2016, ApJ, 822, 53 